\title{
Dynamical Systems with a Cyclic Sign Variation Diminishing Property
}

\author{
Tsuff Ben Avraham, Guy Sharon, Yoram Zarai and Michael Margaliot
}

\begin{abstract}
Several studies analyzed certain nonlinear dynamical systems by showing that the cyclic number of sign variations in the vector of derivatives is an integer-valued Lyapunov function. These results are based on direct analysis of the dynamical equation satisfied by the vector of derivatives, i.e. the variational system. However, it is natural to assume that they follow from the fact that the transition matrix in the variational system satisfies a variation diminishing property (VDP) with respect to the cyclic number of sign variations in a vector. Motivated by this, we develop the theoretical framework of linear time-varying systems whose solution satisfies a VDP with respect to the cyclic number of sign variations. This provides an analogue of the work of Schwarz on totally positive differential systems, i.e. linear time-varying systems whose solution satisfies a VDP with respect to the standard (non-cyclic) number of sign variations.
\end{abstract}

\section{Index Terms}

Totally positive matrices, totally positive differential systems, minor, compound matrices, cooperative dynamical systems, cyclic sign variation diminishing property, stability analysis.

\section{INTRODUCTION}

Let $\mathbb{M}^{+} \subset \mathbb{R}^{n \times n}\left[\mathbb{M} \subset \mathbb{R}^{n \times n}\right]$ denote the subset of $n \times n$ real matrices that are tridiagonal with positive [nonnegative] entries on the super- and sub-diagonals. In an interesting paper, Smillie [24] considered the nonlinear system

$$
\dot{y}=f(y),
$$

with $y(t) \in \mathbb{R}^{n}$, satisfying that its Jacobian $J(y):=\frac{\partial f}{\partial y}(y) \in \mathbb{M}^{+}$for all $y$. He showed that every trajectory of such a system either leaves any compact set or converges to an equilibrium. This result has found many applications as well as several interesting generalizations (see, e.g. [16], [2], [3], [26], [5]). Smillie's analysis is based on showing that the number of sign variations in the vector of derivatives $z(t):=\dot{y}(t)$ can only decrease with time. This was done by direct analysis of the differential equation for $z(t)$, namely, $\dot{z}=J(y) z$.

Recently, it has been shown [17] that Smillie's results are intimately related to the pioneering, yet forgotten, work of Schwarz [23] on linear totally positive differential systems (TPDSs) 1 Recall that a

Research supported in part by research grants from the Israel Science Foundation and the US-Israel Binational Science Foundation.

The authors are with the School of Electrical Engineering, Tel-Aviv University, Tel-Aviv 69978, Israel. Corresponding author: Michael Margaliot (e-mail: michaelmeeng.tau.ac.il)

${ }^{1}$ We use here a slightly different notion than in [23] that agrees with more modern terminology in the field of totally positive and totally nonnegative matrices. 
matrix is called totally positive (TP) [totally nonnegative (TN)] if all its minors are positive [nonegative]. These matrices enjoy a rich and beautiful theory [4], [20]. In particular, multiplying a vector by a TP matrix can only decrease the number of sign variations in the vector.

Schwarz considered the linear time-varying system

$$
\dot{x}(t)=A(t) x(t)
$$

with $A(t)$ a continuous matrix function of $t$. He called this system a TPDS on a time interval $(a, b)$ if its transition matrix $\Phi\left(t, t_{0}\right)$ is totally positive for any pair $\left(t_{0}, t\right)$ with $a<t_{0}<t<b$. Here the transition matrix is the matrix satisfying $x(t)=\Phi\left(t, t_{0}\right) x\left(t_{0}\right)$. In particular, $\Phi\left(t_{0}, t_{0}\right)=I$. In the special case where $A(t)$ is a constant matrix, i.e. $A(t) \equiv A$ then $\Phi\left(t, t_{0}\right)=\exp \left(\left(t-t_{0}\right) A\right)$. Of course, the transition matrix is real, square, and nonsingular.

Schwarz showed that if $A(t)$ is a continuous matrix function of $t$ then a necessary and sufficient condition for TPDS is that $A(t) \in \mathbb{M}$ for all $t \in(a, b)$, and every entry on the sub- or super-diagonal of $A(t)$ is not zero on a time interval. In the particular case of a constant matrix $A(t) \equiv A$ this means that $\dot{x}(t)=A x(t)$ is TPDS if and only if (iff) $A \in \mathbb{M}^{+}$.

Schwarz [23] also showed that if (2) is TPDS then the number of sign changes in $x(t)$ can only decrease with time. To explain this, we recall three definitions for the number of sign variations in a vector. For a vector $y \in \mathbb{R}^{n}$ with no zero entries, let $\sigma(y)$ denote the number of indexes $k \in\{1, \ldots, n-1\}$ such that $y_{k} y_{k+1}<0$. By continuity, it is possible to extend the domain of definition of $\sigma$ to the set

$$
\begin{aligned}
& V:=\left\{y \in \mathbb{R}^{n} \mid y_{1} \neq 0, y_{n} \neq 0,\right. \\
& \left.\quad \text { if } y_{i}=0 \text { for some } 2 \leq i \leq n-1 \text { then } y_{i-1} y_{i+1}<0\right\} .
\end{aligned}
$$

For example $y:=\left[\begin{array}{lll}1 & \varepsilon & -1\end{array}\right]^{\prime} \in V$ and $\sigma(y)=1$ for all $\varepsilon \in \mathbb{R}$ (including $\varepsilon=0$ ). Two more definitions for the number of sign variations in a vector, that are well-defined for any $y \in \mathbb{R}^{n}$, are

$$
s^{-}(y):=\sigma(\bar{y})
$$

where $\bar{y}$ is the vector obtained from $y$ by deleting all zero entries, and

$$
s^{+}(y):=\max _{z \in P(y)}\{\sigma(z)\}
$$

where $P(y)$ is the set of vectors obtained by replacing each zero entry in $y$ by either 1 or -1 . Clearly, $s^{-}(y) \leq$ $s^{+}(y)$ for all $y$. For example, for $y=\left[\begin{array}{lll}0 & 1 & -2\end{array}\right]^{\prime}, s^{-}(y)=1$ and $s^{+}(y)=2$. Let

$$
W:=\left\{y \in \mathbb{R}^{n} \mid s^{-}(y)=s^{+}(y)\right\} .
$$

It is straightforward to show that $W=V$.

A classical and important result from the theory of TP matrices [4] states that if $A$ is TP then

$$
s^{+}(A x) \leq s^{-}(x), \quad \text { for all } x \neq 0 \text {. }
$$

At this point we can already see the connection between this sign variation diminishing property (VDP) 
of TP matrices and the work of Smillie. Indeed, recall that $z:=\dot{y}=f(y)$, and thus,

$$
\dot{z}=\frac{\partial f}{\partial y}(y) \dot{y}=J(y) z .
$$

This is the variational system associated with (1). The assumptions of Smillie on the structure of $J$ imply that (4) is a TPDS and thus for any $t>t_{0}$,

$$
\begin{aligned}
s^{+}(z(t)) & =s^{+}\left(\Phi\left(t, t_{0}\right) z\left(t_{0}\right)\right) \\
& \leq s^{-}\left(z\left(t_{0}\right)\right) .
\end{aligned}
$$

From this it follows that $\sigma(z(t)) \in V$ for all $t$, except perhaps for up to $n-1$ time points $t_{i}$, and that at these points

$$
\sigma\left(z\left(t_{i}^{+}\right)\right)<\sigma\left(z\left(t_{i}^{-}\right)\right)
$$

see e.g. [23], [17].

As briefly mentioned in [17], several papers analyzed various properties of nonlinear dynamical systems by showing that the number of cyclic sign variations in the vector of derivatives $z(t)$ is nonincreasing with time (see, e.g., [7], [25], [15]). These results were proven directly, but it is natural to speculate that they are related to the fact that the transition matrix of the variational system satisfies a cyclic variation diminishing property (CVDP). In this paper, we develop the theoretical framework of such systems. We say that the linear time-varying system $\dot{x}(t)=A(t) x(t)$ is a cyclic variation diminishing differential system (CVDDS) if its transition matrix cannot increase the number of cyclic sign variations. This is the "cyclic analogue" of a TPDS.

The first step is to address the following question: when does multiplication by a matrix $A$ cannot increase the cyclic number of sign variations in a vector? Schoenberg and Whitney [22] already addressed this question and subsequent work of Karlin [12, Ch. 5] includes important characterizations of such matrices (and, more generally, kernels). However, the emphasis is on matrices $A \in \mathbb{R}^{n \times m}$, with $n>m$, whereas for the case of dynamical systems the relevant case is square and nonsingular transition matrices. We provide a simple necessary and sufficient condition for a nonsingular square matrix $A$ to satisfy a CVDP (see Thm. 2 below).

The next step is to consider the matrix differential equation $\dot{\Phi}(t)=A(t) \Phi(t)$. For a constant matrix $A$, we provide a necessary and sufficient condition for the transition matrix $\exp \left(\left(t-t_{0}\right) A\right)$, with $t>t_{0}$, to satisfy a CVDP (Thm. 5). We then extend this to the case where $A(t)$ is continuous in $t$ (Thm. 6). In the more general case where $t \rightarrow A(t)$ is measurable (but not necessarily continuous) we provide a sufficient condition for the transition matrix to satisfy a CVDP (Thm. 7). We also describe the implications of CVDDS to the solution of the vector equation $\dot{x}=A x$ (Thm. 4).

The remainder of this paper is organized as follows. The next section reviews known definitions and results from the theory of TP matrices that will be used later on. Section III describes our main results. We demonstrate one application of these results to a nonlinear model called the ribosome flow model on a ring. Section $I V$ concludes and discusses directions for future research.

We use standard notation. Vectors [matrices] are denoted by small [capital] letters. $\mathbb{R}^{n}$ is the set of vectors with $n$ real coordinates. For a (column) vector $x \in \mathbb{R}^{n}, x_{i}$ is the $i$ th entry of $x$, and $x^{\prime}$ is the 
transpose of $x$. For a matrix $A, \operatorname{tr}(A)$ denotes the trace of $A$. A square matrix $B$ is called Metzler if every off-diagonal entry of $B$ is nonnegative. The square identity matrix is denoted by $I$, with dimension that should be clear from context.

\section{PRELIMinaRies}

We briefly review known definitions and results from the rich and beautiful theory of totally nonnegative and totally positive matrices that will be used later on. For more information and proofs we refer to the excellent monographs [4], [20], [8]. Unfortunately, this field suffers from nonuniform terminology. We follow the more modern terminology as in [4].

We begin with some notation for the minors of a matrix $A \in \mathbb{R}^{n \times m}$. Pick $r \in\{1, \ldots, \min \{n, m\}\}$, and let $\alpha[\beta]$ denote a set of $r$ integers $1 \leq i_{1}<\cdots<i_{r} \leq n\left[1 \leq j_{1}<\cdots<j_{r} \leq m\right]$. Then the minor of $A$ corresponding to the rows indexed by $\alpha$ and columns indexed by $\beta$ is denoted $A(\alpha \mid \beta)$. For example, for $A=\left[\begin{array}{lll}1 & 2 & 3 \\ 4 & 5 & 6 \\ 7 & 8 & 9\end{array}\right], \alpha=\{1,3\}$ and $\beta=\{1,2\}, A(\alpha \mid \beta)=\operatorname{det}\left(\left[\begin{array}{ll}1 & 2 \\ 7 & 8\end{array}\right]\right)=-6$.

Pick $A \in \mathbb{R}^{n \times m}, B \in \mathbb{R}^{m \times p}$, and let $C:=A B$. The Cauchy-Binet formula [4, Ch. 1] asserts that for any two sets $\alpha \subseteq\{1, \ldots, n\}, \beta \subseteq\{1, \ldots, p\}$ with the same cardinality $k \in\{1, \ldots, \min \{n, m, p\}\}$,

$$
C(\alpha \mid \beta)=\sum_{|\gamma|=k} A(\alpha \mid \gamma) B(\gamma \mid \beta)
$$

Here the sum is over all $\gamma=\left\{i_{1}, \ldots, i_{k}\right\}$, with $1 \leq i_{1}<\cdots<i_{k} \leq m$. Thus, every minor of $A B$ is the sum of products of minors of $A$ and $B$. For example, for $n=m=p$ and $k=n$ Eq. (5) gives the well-known formula $\operatorname{det}(A B)=\operatorname{det}(A) \operatorname{det}(B)$.

We now turn to review the VDPs of TP matrices or, more generally, of sign-regular matrices.

Definition 1. A matrix $A \in \mathbb{R}^{n \times m}$ is called sign-regular of order $k$ (denoted $S R_{k}$ ) if all minors of order $k$ have the same non-strict sign. It is called strictly sign-regular of order $k\left(S S R_{k}\right)$ if all its minors of order $k$ are non-zero and have the same sign. It is called strictly sign-regular (SSR) if it is $S S R_{k}$ for all $k \in\{1, \ldots, \min \{n, m\}\}$, that is, all its minors of a given size are non-zero and share a common sign (that may vary from size to size).

For example, the matrix $\left[\begin{array}{ll}1 & 2 \\ 0 & 0\end{array}\right]$ is $S R_{1}$ and $S R_{2}$, and $\left[\begin{array}{ll}1 & 2 \\ 3 & 1\end{array}\right]$ is SSR.

Clearly, a TP matrix is SSR. A classical result (see, e.g. [8, Ch. V]) states that a matrix $A \in \mathbb{R}^{n \times m}$, with $n>m$, is SSR iff it satisfies the strong sign variation diminishing property (SVDP):

$$
s^{+}(A x) \leq s^{-}(x), \quad \text { for all } x \in \mathbb{R}^{m} \backslash\{0\} .
$$

Similarly, a square and nonsingular matrix $A \in \mathbb{R}^{n \times n}$ is SSR iff it satisfies the SVDP. Note that this is not true for singular matrices. For example, it is straightforward to verify that $A=\left[\begin{array}{ll}2 & 2 \\ 1 & 1\end{array}\right]$ satisfies the SVDP, but it is not SSR.

When using sign-regular matrices to study dynamical systems, it is important to bear in mind that in general the signs of minors are not invariant under similarity transformations. An important exception 
is positive diagonal scaling. Indeed, if $D$ is a diagonal matrix with positive entries on the diagonal then multiplying a matrix $A$ by $D$ either on the left or right changes the sign of no minor of $A$, so in particular $D A D^{-1}$ is SR [SSR] if and only if $A$ is SR [SSR].

Our first goal is to characterize square and nonsingular matrices that satisfy an SVDP with respect to the cyclic number of sign variations.

\section{A. Cyclic number of sign variations}

For $y \in \mathbb{R}^{n}$, let

$$
s_{c}^{-}(y):=\max _{i \in\{1, \ldots, n\}} s^{-}\left(\left[\begin{array}{llllll}
y_{i} & \ldots & y_{n} & y_{1} & \ldots & y_{i}
\end{array}\right]^{\prime}\right),
$$

This can be explained as follows: place the entries of $y$ along a circular ring so that $y_{n}$ is followed by $y_{1}$, then count $s^{-}$starting from any entry along the ring, and find the maximal value.

For example, for $y=\left[\begin{array}{llll}0 & 1 & 0 & -3\end{array}\right]^{\prime}, s_{c}^{-}(y)=s^{-}\left(\left[\begin{array}{lllll}1 & 0 & -3 & 0 & 1\end{array}\right]^{\prime}\right)=2$. Similarly,

$$
s_{c}^{+}(y):=\max _{i \in\{1, \ldots, n\}} s^{+}\left(\left[\begin{array}{llllll}
y_{i} & \ldots & y_{n} & y_{1} & \ldots & y_{i}
\end{array}\right]^{\prime}\right),
$$

but here if $y_{i}=0$ then in the calculation of $s^{+}\left(\left[\begin{array}{llllll}y_{i} & \ldots & y_{n} & y_{1} & \ldots & y_{i}\end{array}\right]^{\prime}\right)$ both $y_{i}$ s are replaced by either 1 or -1 . For example, for $y=\left[\begin{array}{llll}0 & 1 & 0 & -3\end{array}\right]^{\prime}, s_{c}^{+}(y)=s^{+}\left(\left[\begin{array}{lllll}1 & 0 & -3 & 0 & 1\end{array}\right]^{\prime}\right)=2$. Note that $s_{c}^{-}(y) \leq s_{c}^{+}(y)$ for all $y \in \mathbb{R}^{n}$, and that both $s_{c}^{-}(y), s_{c}^{+}(y)$ are invariant under cyclic shifts of the vector $y$.

There is a simple and useful relation between the non-cyclic and cyclic number of sign variations of a vector.

Lemma 1. For any vector $x$,

$$
s_{c}^{-}(x)= \begin{cases}s^{-}(x), & \text { if } s^{-}(x) \text { is even } \\ s^{-}(x)+1, & \text { if } s^{-}(x) \text { is odd }\end{cases}
$$

and, similarly,

$$
s_{c}^{+}(x)= \begin{cases}s^{+}(x), & \text { if } s^{+}(x) \text { is even }, \\ s^{+}(x)+1, & \text { if } s^{+}(x) \text { is odd }\end{cases}
$$

For the sake of completeness, we include a proof of this result.

Proof of Lemma 1, Pick $x \in \mathbb{R}^{n}$. If $x=0$ then clearly $s^{-}(x)=s_{c}^{-}(x)=0$, and $s^{+}(x)=n-1$, furthermore, if $n$ is even then $s_{c}^{+}(x)=n$, and if $n$ is odd then $s_{c}^{+}(x)=n-1$. Thus, in this case Lemma 1 holds.

Now consider the case where $x \in \mathbb{R}^{n} \backslash\{0\}$. Let $p:=s^{-}(x)$. We may assume that the first non-zero entry of $x$ is positive. Then the entries of $x$ can be divided into $p+1$ groups: $\left(x_{1}, \ldots, x_{v_{1}}\right),\left(x_{v_{1}+1}, x_{v_{1}+2}, \ldots, x_{v_{2}}\right)$, $\ldots,\left(x_{v_{p}+1}, x_{v_{p}+2}, \ldots, x_{v_{p+1}}\right)$, where $x_{1}, \ldots, x_{v_{1}} \geq 0$ (with at least one of these entries positive), $x_{v_{1}+1}<0$, $x_{v_{1}+2}, \ldots, x_{v_{2}} \leq 0, x_{v_{2}+1}>0$, and so on. If $p$ is even then $x_{v_{p}+1}>0$ and $x_{v_{p}+2}, \ldots, x_{v_{p+1}} \geq 0$. Thus, 
the signs of the first and last group agree and (6) yields $s_{c}^{-}(x)=s^{-}(x)$. If $p$ is odd then $x_{v_{p}+1}<0$ and $x_{v_{p}+2}, \ldots, x_{v_{p+1}} \leq 0$, so $s_{c}^{-}(x)=s^{-}(x)+1$. The proof for $s_{c}^{+}(x)$ is similar.

Note that Lemma 1 implies in particular that $s_{c}^{-}(x), s_{c}^{+}(x)$ is always an even number. Furthermore, for $x \in \mathbb{R}^{n}, s^{-}(x), s^{+}(x)$ take values in $\{0,1, \ldots, n-1\}$, so we conclude that

$$
s_{c}^{-}(x), s_{c}^{+}(x) \in \begin{cases}\{0,2,4, \ldots, n\}, & \text { if } n \text { is even } \\ \{0,2,4, \ldots, n-1\}, & \text { if } n \text { is odd }\end{cases}
$$

We now consider the relation between non-cyclic and cyclic VDPs. Suppose that $A \in \mathbb{R}^{n \times m}$ and $x \in \mathbb{R}^{m}$ satisfy

$$
s^{+}(A x) \leq s^{-}(x) .
$$

If $s^{+}(A x)$ is even then $s_{c}^{+}(A x)=s^{+}(A x) \leq s^{-}(x) \leq s_{c}^{-}(x)$. If $s^{+}(A x)$ is odd and $s^{-}(x)$ is odd then $s_{c}^{+}(A x)=s^{+}(A x)+1 \leq s^{-}(x)+1=s_{c}^{-}(x)$. If $s^{+}(A x)$ is odd and $s^{-}(x)$ is even then (8) gives $s^{+}(A x)+1 \leq s^{-}(x)$, so $s_{c}^{+}(A x)=s^{+}(A x)+1 \leq s^{-}(x)=s_{c}^{-}(x)$. In all cases we see that (8) implies that

$$
s_{c}^{+}(A x) \leq s_{c}^{-}(x)
$$

We conclude that if $A$ satisfies a VDP with respect to (w.r.t.) a non-cyclic number of sign variations it also satisfies the same VDP w.r.t. the cyclic number.

However, it turns out that a weaker property is enough. To show this consider a matrix $A \in \mathbb{R}^{n \times n}$ that is SSR. Then $s^{+}(A x) \leq s^{-}(x)$ for all $x \in \mathbb{R}^{n} \backslash\{0\}$, i.e. $A$ satisfies the SVDP. As noted above, this means that $A$ also satisfies the strong cyclic VDP (SCVDP):

$$
s_{c}^{+}(A x) \leq s_{c}^{-}(x), \quad \text { for all } x \in \mathbb{R}^{n} \backslash\{0\} .
$$

Let $P_{1}, P_{2} \in \mathbb{R}^{n \times n}$ be cyclic permutation matrices. Since $s_{c}^{+}(x), s_{c}^{-}(x)$ are invariant under cyclic permutations of $x$, we have that for all $z \in \mathbb{R}^{n} \backslash\{0\}$,

$$
\begin{aligned}
s_{c}^{+}\left(P_{1} A P_{2}^{-1} P_{2} z\right) & =s_{c}^{+}(A z) \\
& \leq s_{c}^{-}(z) \\
& =s_{c}^{-}\left(P_{2} z\right)
\end{aligned}
$$

Thus, we conclude that $B:=P_{1} A P_{2}^{-1}$ also satisfies the SCVDP, but $B$ does not necessarily satisfy the SVDP. The next example demonstrates this.

Example 1. For $n=3$, consider the matrix $A:=\left[\begin{array}{lll}5 & 4 & 1 \\ 4 & 6 & 4 \\ 1 & 4 & 5\end{array}\right]$. This matrix is TP and thus satisfies both the SVDP and the SCVDP. Let $P_{1}:=\left[\begin{array}{lll}0 & 1 & 0 \\ 0 & 0 & 1 \\ 1 & 0 & 0\end{array}\right]$ and $P_{2}:=I$. Then $B:=P_{1} A P_{2}^{-1}=\left[\begin{array}{lll}4 & 6 & 4 \\ 1 & 4 & 5 \\ 5 & 4 & 1\end{array}\right]$. This matrix satisfies the SCVDP. However, B is not SSR (it has both positive and negative minors of order 2), and thus it does not satisfy the SVDP. 
Before ending this section, we state a well-known and important result that will be used later on.

Proposition 1. Consider a set of $m$ vectors $u^{1}, \ldots, u^{m} \in \mathbb{R}^{n}$, with $m<n$. Define the matrix $U \in \mathbb{R}^{n \times m}$ by

$$
U:=\left[\begin{array}{llll}
u^{1} & u^{2} & \ldots & u^{m}
\end{array}\right]
$$

The following two conditions are equivalent:

(1) For any $c_{1}, \ldots, c_{m} \in \mathbb{R}$, that are not all zero,

$$
s^{+}\left(\sum_{k=1}^{m} c_{i} u^{i}\right) \leq m-1 .
$$

(2) The matrix $U$ is $S S R_{m}$.

For a proof, see e.g., [17].

Remark 1. Note that the assumption that $m<n$ cannot be dropped. For example, if $m=n$ then condition (1) always holds, whereas condition (2) holds iff $U$ is nonsingular.

\section{Main Results}

Our first goal is to provide a necessary and sufficient condition for a square nonsingular matrix to satisfy the SCVDP. We begin by stating an auxiliary result that will be used later on.

\section{A. Non-standard VDP}

We derive a necessary and sufficient condition for a square and nonsingular matrix to satisfy a nonstandard VDP. This result seems to be new and may be of independent interest, as it gives for any value $r$ a clear interpretation of the $S S R_{r}$ property in terms of this non-standard VDP.

Theorem 1. Let $A \in \mathbb{R}^{n \times n}$ be a nonsingular matrix. Pick $p \in\{0, \ldots, n-1\}$. Then the following two conditions are equivalent:

(1) For any vector $c \in \mathbb{R}^{n} \backslash\{0\}$ with $s^{-}(c) \leq p$,

$$
s^{+}(A c) \leq p
$$

(2) A is $S S R_{p+1}$.

Example 2. Consider the case $p=0$. If $c \in \mathbb{R}^{n} \backslash\{0\}$ satisfies $s^{-}(c) \leq 0$, i.e. $s^{-}(c)=0$ then we may assume that every entry of $c$ is nonnegative, and at least one entry is positive (as $c \neq 0)$. Then Ac is a nonnegative combination of the columns of $A$ with at least one column taken with a positive weight. Also, $A c \neq 0$, as $A$ is nonsingular.

If condition (2) holds i.e. A is $S S R_{1}$ then we may assume that all entries of $A$ are positive. Thus, every entry of $A c$ is positive, so $s^{+}(A c)=0$ and condition (1) holds.

Suppose that condition (1) holds. Taking $c=e^{k}$, where $e^{k}$ is the kth canonical vector in $\mathbb{R}^{n}$, we get that $s^{+}\left(A e^{k}\right)=0$, i.e. all the entries in column $k$ of $A$ have the same strict sign. Let $\varepsilon_{k} \in\{-1,1\}$ denote this sign. Seeking a contradiction, assume that there exist $i, j$ such that $\varepsilon_{i}=1$ and $\varepsilon_{j}=-1$. Then 
we can find $d_{i}, d_{j}>0$ such that for $c=d_{i} e^{i}+d_{j} e^{j}$ the vector $A c=d_{i} A e^{i}+d_{j} A e^{j}$ includes a zero entry, so $s^{+}(A c)>0$. This contradicts condition (1), so we conclude that all the $\varepsilon_{k} s$ are identical, i.e. $A$ is $S S R_{1}$.

Example 3. Consider the matrix

$$
A=\left[\begin{array}{llll}
1 & 2 & 0 & 0 \\
0 & 1 & 1 & 0 \\
0 & 0 & 2 & 1 \\
1 & 0 & 0 & 2
\end{array}\right] .
$$

It is straightforward to verify that this matrix is nonsingular and $S S R_{3}$, i.e. Condition (2) holds for $p=2$. We will show that Condition (1) holds for this value of $p$. Pick a vector $c \in \mathbb{R}^{4} \backslash\{0\}$ with $s^{-}(c) \leq 2$. Note that

$$
A c=\left[\begin{array}{llll}
c_{1}+2 c_{2} & c_{2}+c_{3} & 2 c_{3}+c_{4} & c_{1}+2 c_{4}
\end{array}\right]^{\prime} .
$$

Seeking a contradiction, assume that $s^{+}(A c)>2$, i.e. $s^{+}(A c)=3$. Then without loss of generality we can assume that

$$
\begin{aligned}
c_{1}+2 c_{2} & \geq 0, \\
c_{2}+c_{3} & \leq 0, \\
2 c_{3}+c_{4} & \geq 0, \\
c_{1}+2 c_{4} & \leq 0 .
\end{aligned}
$$

We consider three cases.

Case 1. Suppose that $c_{1}<0$. Then the first equation in (12) yields $c_{2}>0$, the second equation gives $c_{3}<0$, and the third yields $c_{4}>0$. But this means that $s^{-}(c)=3$ and this is a contradiction.

Case 2. Suppose that $c_{1}=0$. Then the first three equations in (12) yield $c_{2} \geq 0, c_{3} \leq 0$, and $c_{4} \geq 0$. Now the fourth equation gives $c_{4}=0$. Substituting this in the third equation gives $c_{3}=0$, and the second equation gives $c_{2}=0$. We conclude that $c=0$, which is a contradiction.

Case 3. Suppose that $c_{1}>0$. Then the fourth equation in (12) yields $c_{4}<0$, the third gives $c_{3}>0$, and the second gives $c_{2}<0$. This means that $s^{-}(c)=3$, which is a contradiction.

Summarizing, in each case we obtained a contradiction to $s^{+}(A c)=3$, so $s^{+}(A c) \leq 2$.

We emphasize that condition (1) in Thm. 1 does not assert that $s^{-}(c) \leq p$ implies that $s^{+}(A c) \leq s^{-}(c)$, but only that $s^{+}(A c) \leq p$. For example, for the matrix $A$ in (11) and the vector $c=\left[\begin{array}{llll}0 & 0 & 0 & -1\end{array}\right]^{\prime}$ we have $s^{-}(c)=0 \leq 2$, and $s^{+}(A c)=s^{+}\left(\left[\begin{array}{llll}0 & 0 & -1 & -2\end{array}\right]^{\prime}\right)=2$.

Proof of Thm. 1. First note that for $p=n-1$ both conditions (1) and (2) in Thm. 11hold, as $s^{+}(z) \leq n-1$ for all $z \in \mathbb{R}^{n}$, and $A$ is nonsingular and thus $S S R_{n}$. Also, we already proved Thm. 1 when $p=0$. Thus, we need to prove the result for any $p \in\{1, \ldots, n-2\}$.

Assume that condition (1) holds for some value $p$ in the range $\{1, \ldots, n-2\}$. We will show that all minors of order $p+1$ of $A$ are non-zero and have the same sign. Pick a set of $p+2$ indices $1 \leq k_{1}<$ 
$k_{2}<\cdots<k_{p+2} \leq n$. For any $c \in \mathbb{R}^{n} \backslash\{0\}$ with $s^{-}(c) \leq p$ define $\bar{c} \in \mathbb{R}^{n}$ by:

$$
\bar{c}_{i}:= \begin{cases}c_{i}, & \text { if } i \in\left\{k_{1}, \ldots, k_{p+1}\right\}, \\ 0, & \text { otherwise. }\end{cases}
$$

Then $s^{-}(\bar{c}) \leq p$, as $\bar{c}$ has no more than $p+1$ non-zero entries. Let $a^{i} \in \mathbb{R}^{n}$ denote the $i$ th column of $A$. Then $A \bar{c}=\sum_{i=1}^{n} \bar{c}_{i} a^{i}=\sum_{j=1}^{p+1} c_{k_{j}} a^{k_{j}}$. Applying condition (1) implies that for any $\bar{c} \neq 0, s^{+}(A \bar{c}) \leq p$. This means that the set $\left\{a^{k_{1}}, \ldots, a^{k_{p+1}}\right\}$ satisfies condition (1) in Prop. 1 (note that $n>p+1$ ). Thus, all minors of the form

$$
A\left(i_{1} \ldots i_{p+1} \mid k_{1} \ldots k_{p+1}\right)
$$

with $1 \leq i_{1}<i_{2}<\cdots<i_{p+1} \leq n$, are non-zero and have the same sign. Denote this sign by $\varepsilon\left(k_{1}, \ldots, k_{p+1}\right) \in$ $\{-1,1\}$. It remains to show that this sign depends on the value $p$, but not on the particular choice of $k_{1}, \ldots, k_{p+1}$. Pick $v \in\{1, \ldots, p+1\}$. We will show that

$$
\begin{aligned}
& \varepsilon\left(k_{1}, \ldots, k_{v-1}, k_{v+1}, \ldots, k_{p+2}\right) \\
& =\varepsilon\left(k_{1}, \ldots, k_{v}, k_{v+2}, \ldots, k_{p+2}\right) .
\end{aligned}
$$

To do this, define $p+1$ vectors $\left\{\bar{a}^{k_{1}}, \ldots, \bar{a}^{k_{v}}, \bar{a}^{k_{v+2}}, \ldots, \bar{a}^{k_{p+2}}\right\}$ by

$$
\bar{a}^{k_{i}}:= \begin{cases}a^{k_{i}}, & i \neq v, \\ d_{v} a^{k_{v}}+d_{v+1} a^{k_{v+1}}, & i=v,\end{cases}
$$

where $d_{v}, d_{v+1}>0$. Pick scalars $\bar{c}_{1}, \ldots, \bar{c}_{v}, \bar{c}_{v+2}, \ldots, \bar{c}_{p+2}$, that are not all zero, and let

$$
a:=\sum_{\substack{i=1 \\ i \neq v+1}}^{p+2} \bar{c}_{i} \bar{a}^{k_{i}} .
$$

Then

$$
a=\sum_{i=1}^{p+2} g_{k} a^{k_{i}}
$$

with $g_{v}:=\bar{c}_{v} d_{v}, g_{v+1}:=\bar{c}_{v} d_{v+1}$, and $g_{i}=\bar{c}_{i}$ for all other $i$. Let $g:=\left[\begin{array}{lll}g_{1} & \ldots & g_{p+2}\end{array}\right]^{\prime}$. Note that $g \neq 0$, and that since $g_{v} g_{v+1}=\left(\bar{c}_{v}\right)^{2} d_{v} d_{v+1} \geq 0, s^{-}(g) \leq p$. Applying condition (1) to (16) yields $s^{+}(a) \leq p$, that is, $s^{+}\left(\sum_{\substack{i=1 \\ i \neq v+1}}^{p+2} \bar{c}_{i} \bar{a}^{k_{i}}\right) \leq p$. Let $\bar{A} \in \mathbb{R}^{n \times(p+1)}$ be the matrix

$$
\bar{A}:=\left[\begin{array}{lllllll}
\bar{a}^{k_{1}} & \ldots & \bar{a}^{k_{v-1}} & \bar{a}^{k_{v}} & \bar{a}^{k_{v+2}} & \ldots & \bar{a}^{k_{p+2}}
\end{array}\right] .
$$

Applying Prop. 1 to the set of $p+1$ vectors $\bar{a}^{k_{1}}, \ldots, \bar{a}^{k_{v}}, \bar{a}^{k_{v+2}}, \ldots, \bar{a}^{k_{p+2}} \in \mathbb{R}^{n}$ we conclude that all minors

$$
\bar{A}\left(i_{1}, \ldots, i_{p+1} \mid k_{1}, \ldots, k_{v}, k_{v+2}, \ldots, k_{p+2}\right)
$$


are non-zero. Thus, all minors of the form

$$
\begin{aligned}
& d_{v} A\left(i_{1}, \ldots, i_{p+1} \mid k_{1}, \ldots, k_{v}, k_{v+2}, \ldots, k_{p+2}\right) \\
& +d_{v+1} A\left(i_{1}, \ldots, i_{p+1} \mid k_{1}, \ldots, k_{v-1}, k_{v+1}, \ldots, k_{p+2}\right)
\end{aligned}
$$

are non-zero. This holds for all $d_{v}, d_{v+1}>0$, so the two minors in (17) have the same sign. Since this is true for all $v \in\{1, \ldots, p+1\}$, we conclude that the sign $\varepsilon\left(k_{1}, \ldots, k_{p+1}\right)$ does not change if we change any one of the indices $k_{i}$, and thus it is independent of the choice of $k_{1}, \ldots, k_{p+1}$. This completes the proof that condition (1) implies condition (2).

To prove the converse implication, assume that $A=\left[\begin{array}{lll}a^{1} & \ldots & a^{n}\end{array}\right]$ is $S S R_{p+1}$ for some $p \in\{1, \ldots, n-$ $2\}$. Pick $c \in \mathbb{R}^{n} \backslash\{0\}$ such that $s^{-}(c) \leq p$. Let $k:=s^{-}(c)$. We may assume that the first non-zero entry of $c$ is positive. Then we can decompose $c$ into $k+1$ groups:

$$
\begin{aligned}
& \left(c_{1}, c_{2}, \ldots, c_{v_{1}}\right),\left(c_{v_{1}+1}, c_{v_{1}+2}, \ldots, c_{v_{2}}\right), \ldots, \\
& \left(c_{v_{k}+1}, c_{v_{k}+2}, \ldots, c_{v_{k+1}}\right)
\end{aligned}
$$

where $c_{1}, \ldots, c_{v_{1}} \geq 0$ (with at least one of these entries positive); $c_{v_{1}+1}<0, c_{v_{1}+2}, \ldots, c_{v_{2}} \leq 0, c_{v_{2}+1}>0$, and so on. Define vectors $u^{1}, \ldots, u^{k+1} \in \mathbb{R}^{n}$ by

$$
u^{1}:=\sum_{j=1}^{v_{1}}\left|c_{j}\right| a^{j}, u^{2}:=\sum_{j=v_{1}+1}^{v_{2}}\left|c_{j}\right| a^{j}, \ldots
$$

Then

$$
\begin{aligned}
A c & =\sum_{j=1}^{n} c_{j} a^{j} \\
& =u^{1}-u^{2}+u^{3}-\cdots+(-1)^{k} u^{k+1} .
\end{aligned}
$$

Note that every $u^{i}$ is a non-negative and non-trivial sum of a consecutive set of $a^{k} \mathrm{~s}$. We now consider two cases.

Case 1. Suppose that $k=p$. Let $U:=\left[\begin{array}{lll}u^{1} & \ldots & u^{k+1}\end{array}\right]$. Note that the dimensions of $U$ are $n \times(p+1)$ with $n>p+1$. Since $A$ is $S S R_{p+1}$, all the minors of order $(p+1)$ of $U$ are non-zero and have the same sign. Applying Prop. 1 to (19) yields $s^{+}(A c) \leq p$.

Case 2. Suppose that $k<p$. We then split the $k+1$ groups in (18) and generate $p+1$ vectors $w^{1}, \ldots, w^{p+1}$ by modifying $u^{1}, \ldots, u^{k+1}$ as follows. Suppose for concreteness that the first group in (18) contains more than one element and that $c_{1}>0$. If $c_{2}>0$ we replace $u^{1}$ by two vectors $w^{1}:=\left|c_{1}\right| a^{1}$ and $w^{2}:=$ $\sum_{j=2}^{v_{1}}\left|c_{j}\right| a^{j}$, and let $w^{3}:=u^{2}, w^{4}:=u^{3}, \ldots, w^{k+2}:=u^{k+1}$. Note that $A c=\left(w^{1}+w^{2}\right)-w^{3}+w^{4}-w^{5}-$ $\cdots+(-1)^{k} w^{k+2}$. If $c_{2}=0$ we replace $u^{1}$ by two vectors $w^{1}:=\left|c_{1}\right| a^{1}$ and $w^{2}:=a^{2}$, and let $w^{3}:=u^{2}$, $w^{4}:=u^{3}, \ldots, w^{k+2}:=u^{k+1}$. Note that $A c=\left(w_{1}+0 * w_{2}\right)-w^{3}+w^{4}-w^{5}-\cdots+(-1)^{k} w^{k+2}$. We can continue this decomposition, if necessary, using the entry $c_{3}$, etc. If the first group in (18) contains only one element then we can follow the same idea using any entry in any group that contains more than a 
single element. In this way we can generate a set $w^{1}, \ldots, w^{p+1}$ such that

$$
A c=\sum_{j=1}^{p+1} d_{i} w^{i},
$$

where the $d_{i}$ s are not all zero, and the matrix $W:=\left[\begin{array}{lll}w^{1} & \ldots & w^{p+1}\end{array}\right] \in \mathbb{R}^{n \times(p+1)}$ is $S S R_{p+1}$ because $A$ is $S S R_{p+1}$. Applying Prop. 1 to (20) yields $s^{+}(A c) \leq p$.

Summarizing we showed in both cases that condition (2) implies that condition (1) holds. This completes the proof of Thm. 1 .

\section{B. Conditions for cyclic VDP}

The main result in this subsection provides a necessary and sufficient condition for a square and nonsingular matrix to satisfy the SCVDP.

Theorem 2. Let $A \in \mathbb{R}^{n \times n}$ be a nonsingular matrix. The following two conditions are equivalent:

(1) For any vector $x \in \mathbb{R}^{n} \backslash\{0\}$,

$$
s_{c}^{+}(A x) \leq s_{c}^{-}(x) .
$$

(2) The matrix $A$ is $S S R_{r}$ for all odd $r$ in the range $r \in\{1, \ldots, n\}$.

Example 4. Suppose that $A \in \mathbb{R}^{3 \times 3}$ is nonsingular. Then $A$ is $S S R_{3}$. Thm. 2 asserts that $A$ satisfies the SCVDP if and only if all the entries of A are either all positive or all negative. Note that this agrees with the results in Example 1

Proof of Theorem 2. Suppose that condition (2) holds. Pick $x \in \mathbb{R}^{n} \backslash\{0\}$. Let $k$ be such that $s_{c}^{-}(x)=2 k$ (recall that $s_{c}^{-}$and $s_{c}^{+}$are always even). If $2 k=n$ then clearly (21) holds, so we may assume that $2 k \leq$ $n-1$.. Since $s_{c}^{-}(x)=2 k, s^{-}(x) \leq 2 k$. Condition (2) implies in particular that $A$ is $S S R_{2 k+1}$, and Thm. 1 yields $s^{+}(A x) \leq 2 k$. Hence $s_{c}^{+}(A x) \leq 2 k=s_{c}^{-}(x)$. This shows that condition (2) implies condition (1).

To prove the converse implication assume that condition (1) holds. Pick an even number $p \in\{0, \ldots, n-$ $1\}$, and a vector $x \in \mathbb{R}^{n} \backslash\{0\}$ such that $s^{-}(x) \leq p$. Condition (1) yields $s_{c}^{+}(A x) \leq p$, so $s^{+}(A x) \leq p$. Thm. 1 implies that $A$ is $S S R_{p+1}$. Since $p$ is an arbitrary even number in $\{0, \ldots, n-1\}$, we conclude that condition (2) holds.

Using a standard continuity argument yields the following condition for weak cyclic VDP.

Theorem 3. Let $A \in \mathbb{R}^{n \times n}$ be a nonsingular matrix. The following two conditions are equivalent:

(1) For any vector $x \in \mathbb{R}^{n} \backslash\{0\}$,

$$
s_{c}^{-}(A x) \leq s_{c}^{-}(x)
$$

(2) The matrix $A$ is $S R_{r}$ for all odd $r$ in the range $r \in\{1, \ldots, n\}$.

Proof of Thm. 3, Suppose that condition (2) holds. For $y \in \mathbb{R}$, let $F(y)$ denote the $n \times n$ matrix whose $i, j$ entry is $\exp \left(-(i-j)^{2} y\right)$. For example, for $n=3$,

$$
F(y)=\left[\begin{array}{ccc}
1 & \exp (-y) & \exp (-4 y) \\
\exp (-y) & 1 & \exp (-y) \\
\exp (-4 y) & \exp (-y) & 1
\end{array}\right]
$$


It is well-known that $F(y)$ is TP for all $y>0$ [8, Ch. II], and $\lim _{y \rightarrow \infty} F(y)=I$. Fix $y>0$ and let $F:=F(y), B:=F A$. Pick an odd index $r \in\{1, \ldots, n\}$, and let $\alpha, \beta$ denote two sets of $r$ integers $1 \leq$ $i_{1}<\cdots<i_{r} \leq n$ and $1 \leq j_{1}<\cdots<j_{r} \leq n$, respectively. The Cauchy-Binet formula yields

$$
B(\alpha \mid \beta)=\sum_{\gamma} F(\alpha \mid \gamma) A(\gamma \mid \beta)
$$

where the sum is over all $\gamma=\left\{k_{1}, \ldots, k_{r}\right\}$, with $1 \leq k_{1}<\cdots<k_{r} \leq n$. Using the fact that $F$ is TP, all minors of order $r$ of $A$ have the same non-strict sign and they are not all zero (as $A$ is nonsingular), we conclude that $B$ is $S S R_{r}$. Now Thm. 2 implies that for any $x \in \mathbb{R}^{n} \backslash\{0\}, s_{c}^{+}(F(y) A x) \leq s_{c}^{-}(x)$, so

$$
s_{c}^{-}(F(y) A x) \leq s_{c}^{-}(x) .
$$

Taking $y \rightarrow \infty$ yields (22). Thus, condition (2) implies condition (1).

To prove the converse implication, assume that condition (11) holds. Pick $y>0$. Then $F(y)$ is TP and thus satisfies the SVDP and thus the SCVDP, so for any $x \in \mathbb{R}^{n} \backslash\{0\}$,

$$
s_{c}^{+}(F(y) A x) \leq s_{c}^{-}(A x) .
$$

Combining this with (22) yields

$$
s_{c}^{+}(F(y) A x) \leq s_{c}^{-}(x) .
$$

Now Thm. 2 implies that $F(y) A$ is $S S R_{r}$ for all odd $r$. Taking $y \rightarrow \infty$ we conclude that $A$ is $S R_{r}$ for all odd $r$.

Example 5. Consider the case $A \in \mathbb{R}^{2 \times 2}$. In this case, condition (2) in Thm. 3 holds iff $A$ is $S R_{1}$ i.e. iff all the entries in A have the same non-strict sign. We will show directly that in this case condition (1) in Thm. 3 also holds. Pick $x \in \mathbb{R}^{2} \backslash\{0\}$. We consider two cases (recall that $s_{c}^{-}(x)$ is always even).

Case 1. If $s_{c}^{-}(x)=2$ then clearly (22) holds.

Case 2. Suppose that $s_{c}^{-}(x)=0$. This means that $x_{1}, x_{2}$ have the same non-strict sign. We may assume that $x_{1}, x_{2} \geq 0$. Then both entries of $A x=\left[\begin{array}{l}a_{11} x_{1}+a_{12} x_{2} \\ a_{21} x_{1}+a_{22} x_{2}\end{array}\right]$ have the same non-strict sign, so $s_{c}^{-}(A x)=0$ and again (22) holds.

We now proceed to defining the analogue of a TPDS for the case of the cyclic number of sign variations.

C. Dynamical systems satisfying a cyclic VDP

Consider the linear time-varying system (2). The associated matrix differential equation is

$$
\dot{\Phi}(t)=A(t) \Phi(t)
$$

Let $\Phi\left(t, t_{0}\right)$ denote the solution of this equation satisfying $\Phi\left(t_{0}, t_{0}\right)=I$.

Definition 2. We say that (2) (and also (23) is a cyclic variation diminishing differential system (CVDDS) on a time interval $(a, b)$ if $\Phi\left(t, t_{0}\right)$ satisfies the SCVDP for any pair $\left(t_{0}, t\right)$ with $a<t_{0}<t<b$. 
Example 6. Consider the fixed matrix $A=\left[\begin{array}{ll}a_{11} & a_{12} \\ a_{21} & a_{22}\end{array}\right]$. Then

$$
\begin{aligned}
\Phi\left(t, t_{0}\right) & =\exp \left(\left(t-t_{0}\right) A\right) \\
& =\left[\begin{array}{cc}
1+a_{11} d+o(d) & a_{12} d+o(d) \\
a_{21} d+o(d) & 1+a_{22} d+o(d)
\end{array}\right],
\end{aligned}
$$

where $d:=t-t_{0}$. This implies that the following three statements are equivalent: (1) $a_{12}, a_{21}>0$; (2) there exists $\varepsilon>0$ such that $\exp \left(\left(t-t_{0}\right) A\right)$ is $S S R_{1}$ for all $\left(t-t_{0}\right) \in(0, \varepsilon]$; and (3) $\exp \left(\left(t-t_{0}\right) A\right)$ is $S S R_{1}$ for all $t>t_{0}$.

Combining this with Thm. 2 we conclude that the system $\dot{x}=A x$, with $A \in \mathbb{R}^{2 \times 2}$, is CVDDS on any time interval iff $a_{12}, a_{21}>0$.

The next result describes one implication of CVDDS. Let

$$
V_{c}:=\left\{x \in \mathbb{R}^{n}: s_{c}^{-}(x)=s_{c}^{+}(x)\right\} .
$$

Theorem 4. Suppose that (2) is CVDDS on $(a, b)$. If $x(t)$ is not the trivial solution $x(t) \equiv 0$ then:

(1) $s_{c}^{-}(x(t)), s_{c}^{+}(x(t))$ are non-increasing functions of time on $(a, b)$;

(2) $x(t) \in V_{c}$ for all $t \in(a, b)$, except perhaps for up to $\lfloor n / 2\rfloor$ discrete values of $t$;

(3) there exists a time $T \geq 0$ such that

$$
s_{c}^{-}(x(\tau))=s_{c}^{+}(x(\tau)), \quad \text { for all } \tau \geq T,
$$

and for all such $\tau$ no two consecutive entries (in the cyclic order) of $x$ satisfy

$$
x_{i}(\tau)=x_{i+1}(\tau)=0 .
$$

Proof of Thm. 4. For any $a<t_{0}<t<b$, we have $x(t)=\Phi\left(t, t_{0}\right) x\left(t_{0}\right)$. Since $x\left(t_{0}\right) \neq 0$ and $\Phi\left(t, t_{0}\right)$ satisfies the SCVDP,

$$
s_{c}^{+}(x(t)) \leq s_{c}^{-}\left(x\left(t_{0}\right)\right) .
$$

Thus, $s_{c}^{-}(x(t)) \leq s_{c}^{+}(x(t)) \leq s_{c}^{-}\left(x\left(t_{0}\right)\right)$. If $x\left(t_{0}\right) \in V_{c}$ then $s_{c}^{-}\left(x\left(t_{0}\right)\right)=s_{c}^{+}\left(x\left(t_{0}\right)\right)$, so (25) yields

$$
s_{c}^{+}(x(t)) \leq s_{c}^{+}\left(x\left(t_{0}\right)\right) \text {. }
$$

If $x\left(t_{0}\right) \notin V_{c}$ then $s_{c}^{-}\left(x\left(t_{0}\right)\right)<s_{c}^{+}\left(x\left(t_{0}\right)\right)$, so

$$
s_{c}^{+}(x(t))<s_{c}^{+}\left(x\left(t_{0}\right)\right) .
$$

Thus, $s_{c}^{+}(x(t))$ never increases, and it strictly decreases as $x(t)$ goes through a point that is not in $V_{c}$. Since $s_{c}^{+}(x(t))$ is even the decrease is by at least two. Since $s_{c}^{+}$takes values in $\{0,1, \ldots, n\}[\{0,1, \ldots, n-$ $1\}$ ] when $n$ is even [odd], this implies that $x(t) \in V_{c}$ for all $t$, except perhaps for up to $\lfloor n / 2\rfloor$ discrete points. This proves the first two assertions in the theorem. The third assertion follows immediately from the second and the fact that if $y$ has two consecutive (in the cyclic order) zeros then $s_{c}^{-}(y)<s_{c}^{+}(y)$. 
The proof of Thm. 4 shows that we may view $s^{-}(x(t)), s^{+}(x(t))$ as integer-valued Lyapunov functions of the time-varying linear system (2).

Using Thm. 2 yields a converse for Thm. 4.

Corollary 1. Suppose that the solution of (2) satisfies (25) for all $a<t_{0}<t<b$ and all $x\left(t_{0}\right) \in \mathbb{R}^{n} \backslash\{0\}$. Then for all $\left(t_{0}, t\right)$ with $a<t_{0}<t<b$ all the odd minors of the transition matrix $\Phi\left(t, t_{0}\right)$ are positive.

Proof of Corollary 1, Pick an odd $r \in\{1, \ldots, n\}$. The matrix $\Phi\left(t_{0}, t_{0}\right)=I$ has a minor of order $r$ that is one. By Thm. 2, all minors of order $r$ of $\Phi\left(t, t_{0}\right)$, with $t>t_{0}$, are non-zero and have the same sign. By continuity, this implies that all minors of order $r$ of $\Phi\left(t, t_{0}\right)$, with $t>t_{0}$, are positive. Since $r$ is an arbitrary odd number, this completes the proof.

The next natural question is what conditions on $A(t)$ guarantee that (2) is CVDDS. The next subsection answers this question for the case where $A(t) \equiv A$.

\section{The case A constant}

Definition 3. Let $\mathbb{Q}^{+} \subset \mathbb{R}^{n \times n}$ denote the set of $n \times n$ matrices that are Metzler, irreducible, and can have non-zero entries only on the main diagonal, super- and sub-diagonals and entries $(1, n)$ and $(n, 1)$.

For example, for $n=4$ the matrices:

$$
\left[\begin{array}{cccc}
* & + & 0 & 0 \\
0 & * & + & 0 \\
0 & 0 & * & + \\
+ & 0 & 0 & *
\end{array}\right],\left[\begin{array}{cccc}
* & + & 0 & 0 \\
+ & * & + & 0 \\
0 & + & * & + \\
0 & 0 & + & *
\end{array}\right],
$$

where $*$ denotes some value and + denotes a positive value, are in $\mathbb{Q}^{+}$.

Note that if $A \in \mathbb{Q}^{+}$then $A^{\prime} \in \mathbb{Q}^{+}$, and that if $A, B \in \mathbb{Q}^{+}$then $A+B \in \mathbb{Q}^{+}$.

Theorem 5. Fix a time interval $(a, b)$. Let $A$ be a constant $n \times n$ matrix. The system $\dot{x}=A x$ is $C V D D S$ on $(a, b)$ iff $A \in \mathbb{Q}^{+}$.

Example 7. Consider the case $n=2$. In Example 6 we saw that the system $\dot{x}=A x$, with $A \in \mathbb{R}^{2 \times 2}$, is CVDDS on any time interval iff $a_{12}, a_{21}>0$. On the other-hand, the definition of $\mathbb{Q}^{+}$shows that $A \in \mathbb{Q}^{+}$ iff $a_{12}, a_{21}>0$.

Proving Theorem 5 requires introducing more notation. Before that we make several comments. First, it follows from the definition of $\mathbb{Q}^{+}$that if $A \in \mathbb{M}^{+}$then $A \in \mathbb{Q}^{+}$. This also makes sense, as $A \in \mathbb{M}^{+}$ implies that $\dot{x}=A x$ is TPDS and thus it is also CVDDS. If $A \in\left(\mathbb{Q}^{+} \backslash \mathbb{M}^{+}\right)$then the system is not TPDS and thus $\exp (A t)$ does not satisfy the SVDP. However, the next remark shows that any possible violation of the SVDP has a particular structure.

Remark 2. Pick $A \in\left(\mathbb{Q}^{+} \backslash \mathbb{M}^{+}\right)$. Then $\dot{x}=A x$ is CVDDS, but not TPDS. Suppose that at some time $\tau$ there is an increase in $s^{-}(x(t))$ (the analysis for $s^{+}(x)$ is similar), say,

$$
s^{-}\left(x\left(\tau^{-}\right)\right)=k \text { and } s^{-}\left(x\left(\tau^{+}\right)\right)>k .
$$



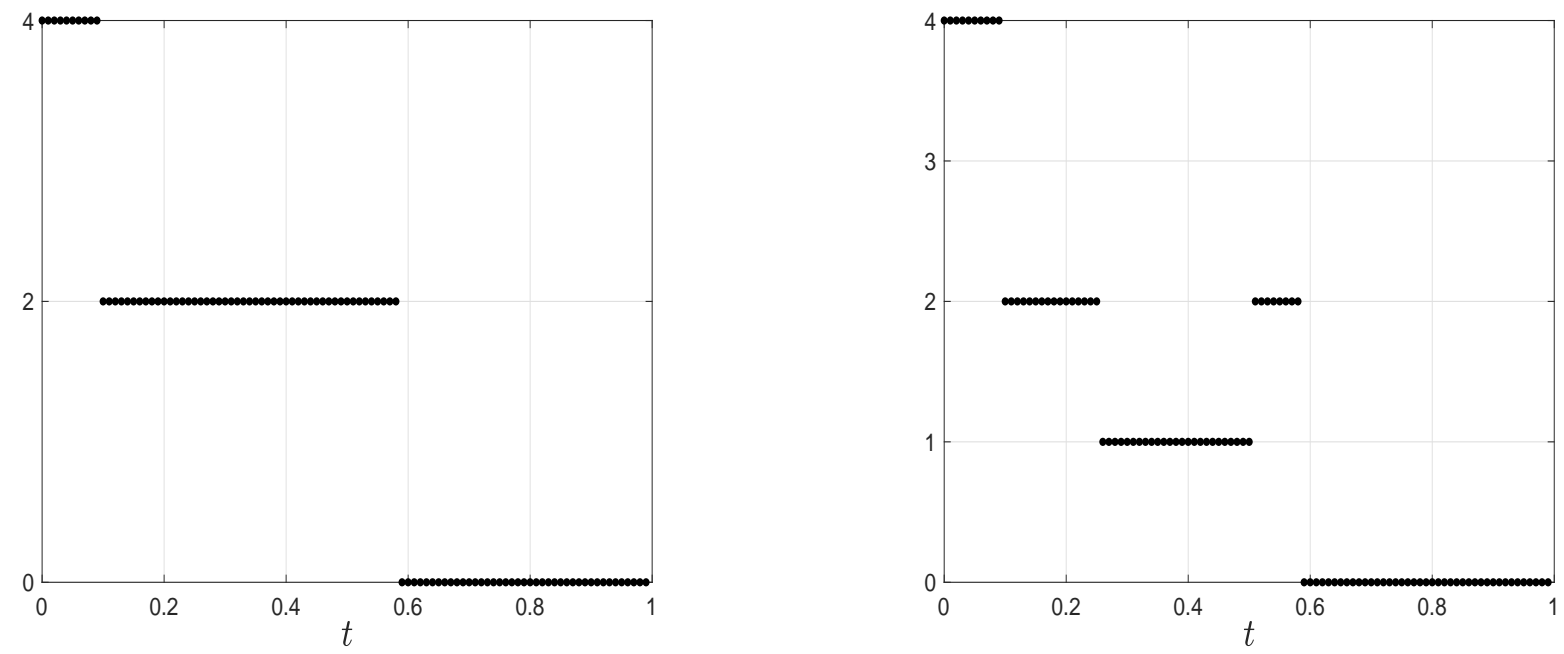

Fig. 1: $s_{c}^{-}(x(t))$ (left) and $s^{-}(x(t))$ (right) as a function of $t \in[0,1]$ for the system in Example 8 ,

Assume that $k$ is even. Then Lemma 1 yields

$$
s_{c}^{-}\left(x\left(\tau^{-}\right)\right)=k \text { and } s_{c}^{-}\left(x\left(\tau^{+}\right)\right)>k,
$$

but this is impossible as the system is CVDDS. We conclude that if (27) holds at some time $\tau$ then $k$ is odd, and by Lemma $\square s_{c}^{-}\left(x\left(\tau^{-}\right)\right)=k+1$. Since the system is CVDDS, we must have $s_{c}^{-}\left(x\left(\tau^{+}\right)\right) \leq k+1$. Lemma 1 now implies that $s^{-}\left(x\left(\tau^{+}\right)\right)=k+1$. Summarizing, if there is an increase in $s^{-}(x(t))$ at time $\tau$ then we must have

$$
\begin{array}{ll}
s^{-}\left(x\left(\tau^{-}\right)\right)=2 i+1, & s^{-}\left(x\left(\tau^{+}\right)\right)=2 i+2, \\
s_{c}^{-}\left(x\left(\tau^{-}\right)\right)=2 i+2, & s_{c}^{-}\left(x\left(\tau^{+}\right)\right)=2 i+2,
\end{array}
$$

for some $i$. In particular, if for some time $T$ we have $s_{c}^{-}(x(t))=s_{c}^{+}(x(t))=0$ for all $t \geq T$ then $s^{-}(x(t))=$ $s^{+}(x(t))=0$ for all $t \geq T$.

Example 8. Consider the system $\dot{x}=A x$, with

$$
A=\left[\begin{array}{ccccc}
-4 & 1 & 0 & 0 & 0 \\
2 & -4 & 4 & 0 & 0 \\
0 & 7 / 2 & -4 & 5 / 2 & 0 \\
0 & 0 & 0 & -4 & 1 \\
5 / 4 & 0 & 0 & 3 / 2 & -4
\end{array}\right]
$$

Note that $A \in\left(\mathbb{Q}^{+} \backslash \mathbb{M}^{+}\right)$, thus the system is CVDDS but not TPDS. Fig. 1 depicts $s_{c}^{-}(x(t))$ and $s^{-}(x(t))$ as a function of $t \in[0,1]$ for the initial condition $x(0)=\left[\begin{array}{lllll}-0.6407 & 1.8089 & -1.0799 & 0.1992 & -1.5210\end{array}\right]^{\prime}$. It may be noticed that $s_{c}^{-}(x(t))$ is piecewise-constant and that at any point where its value changes it decreases by two. On the other-hand $s^{-}(x(t))$ both decreases and increases (as the system is not TPDS), and the increase agrees with the structure described in Remark 2

The proof of Thm.. 5 is based on analyzing the dynamics of all the odd minors of $\Phi\left(t, t_{0}\right)$. To do that, 
we briefly review multiplicative and additive compound matrices (see, e.g., [19], [17]). Given $A \in \mathbb{R}^{n \times n}$ and $p \in\{1, \ldots, n\}$, consider the $\left(\begin{array}{l}n \\ p\end{array}\right)^{2}$ minors of $A$ of size $p \times p$. Recall that each such minor is defined by a set of row indexes $1 \leq i_{1}<i_{2}<\cdots<i_{p} \leq n$ and column indexes $1 \leq j_{1}<j_{2}<\cdots<j_{p} \leq n$, and is denoted by $A(\alpha \mid \beta)$, where $\alpha:=\left\{i_{1}, \ldots, i_{p}\right\}$ and $\beta:=\left\{j_{1}, \ldots, j_{p}\right\}$. The $p$ th multiplicative compound matrix $A^{(p)}$ is the $\left(\begin{array}{l}n \\ p\end{array}\right) \times\left(\begin{array}{l}n \\ p\end{array}\right)$ matrix that includes all these minors ordered lexicographically. The CauchyBinet formula yields

$$
(A B)^{(p)}=A^{(p)} B^{(p)},
$$

justifying the term multiplicative compound.

The $p$ th additive compound matrix of $A$ is defined by

$$
A^{[p]}:=\left.\frac{d}{d h}(I+h A)^{(p)}\right|_{h=0} .
$$

In other words, $A^{[p]}$ is the the term that multiplies $h$ in the Taylor series expansion of $(I+h A)^{(p)}$. Using (30) and (29) gives $(A+B)^{[p]}=A^{[p]}+B^{[p]}$, justifying the term additive compound.

It can be shown [23] that if $\Phi$ satisfies (23) then for any $p \in\{1, \ldots, n\}$,

$$
\frac{d}{d t} \Phi^{(p)}=A^{[p]} \Phi^{(p)}
$$

Thus, the dynamics of $\Phi^{(p)}$, i.e. the dynamics of the minors of order $p$ of $\Phi$, is also a linear system with the matrix $A^{[p]}$.

The matrix $A^{[p]}$ can be determined explicitly. The entry of $A^{[p]}$ corresponding to $(\alpha \mid \beta)=\left(i_{1}, \ldots, i_{p} \mid j_{1}, \ldots, j_{p}\right)$ is:

$$
\begin{cases}\sum_{k=1}^{p} a_{i_{k} i_{k}}, & \text { if }|\alpha \cap \beta|=p \\ (-1)^{\ell+m} a_{i_{\ell} j_{m}}, & \text { if }|\alpha \cap \beta|=p-1 \text { and } i_{\ell} \neq j_{m} \\ 0, & \text { otherwise. }\end{cases}
$$

The first line in (32) corresponds to the case where $i_{k}=j_{k}$ for all $k=1, \ldots, p$, i.e. to the diagonal entries of $A^{[p]}$. The second line describes the case where all the indexes in $\alpha$ and $\beta$ coincide, except for a single index $i_{\ell} \neq j_{m}$. Eq. (32) is usually proven by manipulating determinants [23] or using exterior powers [6].

For example, consider the case $A=\left\{a_{i j}\right\}_{i, j=1}^{4}$. Then (32) yields $A^{[1]}=A$,

$$
\begin{aligned}
& A^{[2]}=\left[\begin{array}{cccccc}
a_{11}+a_{22} & a_{23} & a_{24} & -a_{13} & -a_{14} & 0 \\
a_{32} & a_{11}+a_{33} & a_{34} & a_{12} & 0 & -a_{14} \\
a_{42} & a_{43} & a_{11}+a_{44} & 0 & a_{12} & a_{13} \\
-a_{31} & a_{21} & 0 & a_{22}+a_{33} & a_{34} & -a_{24} \\
-a_{41} & 0 & a_{21} & a_{43} & a_{22}+a_{44} & a_{23} \\
0 & -a_{41} & a_{31} & -a_{42} & a_{32} & a_{33}+a_{44}
\end{array}\right] \\
& A^{[3]}=\left[\begin{array}{cccc}
a_{11}+a_{22}+a_{33} & a_{34} & -a_{24} & a_{14} \\
a_{43} & a_{11}+a_{22}+a_{44} & a_{23} & -a_{13} \\
-a_{42} & a_{32} & a_{11}+a_{33}+a_{44} & a_{12} \\
a_{41} & -a_{31} & a_{21} & a_{22}+a_{33}+a_{44}
\end{array}\right] \text {, }
\end{aligned}
$$


and $A^{[4]}=\operatorname{tr}(A)$.

Proof of Thm. 5. Suppose that

$$
A \in \mathbb{Q}^{+}
$$

We will show that for any odd $p$ the matrix $A^{[p]}$ is Metzler and irreducible. For $p=1$ this is immediate, as $A^{[1]}=A$.

Pick an odd $p \in\{3, \ldots, n\}$. By (32), every off-diagonal entry of $A^{[p]}$ is either zero or has the form $(-1)^{\ell+m} a_{i_{\ell} j_{m}}$. Since $A \in \mathbb{Q}^{+}, A^{[p]}$ can have a non-zero off-diagonal entry only in the following three cases.

Case 1 . The entry $a_{i_{\ell} j_{m}}$ is on the super- or sub-diagonal of $A$. Then $\left\{i_{\ell}, j_{m}\right\}=\{k, k+1\}$ for some $k \in$ $\{1, \ldots, n-1\}$. This means that $\alpha$ and $\beta$ share $p-1$ common indexes, and one of $\{k, k+1\}$ is in $\alpha$ (and not in $\beta$ ) and the other in $\beta$ (and not in $\alpha$ ). This implies that $\ell=m$, and thus $(-1)^{\ell+m} a_{i_{\ell} j_{m}}=(-1)^{2 \ell} a_{i_{\ell} j_{\ell}}$. Since $i_{\ell} \neq j_{\ell}$ and $A$ is Metzler, we conclude that $(-1)^{\ell+m} a_{i_{\ell} j_{m}} \geq 0$.

Case 2. The entry $a_{i_{\ell} j_{m}}$ is entry $(1, n)$ in $A$. This means that $\ell=1, m=p$, so $(-1)^{\ell+m} a_{i_{\ell} j_{m}}=$ $(-1)^{p+1} a_{1 n} \geq 0$, as $p$ is odd.

Case 3. The entry $a_{i_{\ell} j_{m}}$ is entry $(n, 1)$ in $A$. Then $\ell=p, m=1$, so $(-1)^{\ell+m} a_{i_{\ell} j_{m}}=(-1)^{1+p} a_{n 1} \geq 0$.

We conclude that $A^{[p]}$ is Metzler. We now show that $(35)$ implies that $A^{[p]}$ is irreducible. We introduce some notation. Let $G^{[p]}$ denote the adjacency graph associated with the matrix $A^{[p]}$. Every node in this graph corresponds to a set of $p$ indexes $1 \leq i_{1}<i_{2}<\cdots<i_{p} \leq n$, and there are $\left(\begin{array}{l}n \\ p\end{array}\right)$ nodes. There is a directed edge from node $\alpha=\left\{i_{1}, \ldots, i_{p}\right\}$ to $\beta=\left\{j_{1}, \ldots, j_{p}\right\}$ if exactly $p-1$ entries of $\alpha$ and $\beta$ coincide, the two remaining indexes are $i_{\ell} \neq j_{m}$, and $a_{i_{\ell} j_{m}} \neq 0$. We write $\alpha \leq \beta$ if $i_{k} \leq j_{k}$ for all $k \in\{1, \ldots, p\}$. We write $\alpha \rightsquigarrow \beta$ if there is a path in $G^{[p]}$ starting at $\alpha$ and ending at $\beta$.

We consider two cases.

Case 1. Suppose that all the entries on the sub- and super-diagonals of $A$ are positive. It follows from the results of Schwarz [23], that considered the case $A \in \mathbb{M}^{+}$, that in this case $G^{[k]}$ is strongly connected for all $k$, so $A^{[k]}$ is irreducible for all $k$. In particular, $A^{[p]}$ is irreducible.

Case 2. Suppose that $A \in \mathbb{Q}^{+}$, but there exist $i, j$, with $|i-j|=1$ such that $a_{i j}=0$. We may assume that for some $i \in\{2, \ldots, n\}$ entry $(i, i-1)$ of $A$ is zero. We know that $A$ is irreducible, and using the structure of $\mathbb{Q}^{+}$this implies that

$$
a_{12}>0, a_{23}>0, \ldots, a_{n-1 n}>0, a_{n 1}>0
$$

Let

$$
\begin{aligned}
& \underline{\gamma}:=\{1,2, \ldots, p\}, \\
& \bar{\gamma}:=\{n-p+1, n-p+2, \ldots, n-1, n\},
\end{aligned}
$$

i.e. the lowest and highest nodes in the lexicographic ordering, respectively. It follows from (32) and (36) that for any two nodes $\alpha \neq \beta$ satisfying $\alpha \leq \beta$, we have $\alpha \rightsquigarrow \beta$. In particular, there is a path from $\underline{\gamma}$ to any node, and there is a path from any node to $\bar{\gamma}$. 
Since $a_{n 1}>0$, the edges

$$
\left\{i_{1}, i_{2}, \ldots, i_{p-1}, n\right\} \rightarrow\left\{1, i_{1}, i_{2}, \ldots, i_{p-1}\right\}
$$

with $2 \leq i_{1}<i_{2}<\cdots<i_{p-1}<n$ are included in $G^{[p]}$. In particular, this includes the edges $\bar{\gamma} \rightarrow \delta:=$ $\{1, n-p+1, n-p+2, \ldots, n-2, n-1\}$, and $\zeta:=\{2,3, \ldots, p, n\} \rightarrow \underline{\gamma}$.

Now pick two distinct nodes $\alpha, \beta$. We will show that $\alpha \rightsquigarrow \beta$. If

$$
p \geq n-2
$$

then $\delta \leq \zeta$, so $G^{[p]}$ includes the path:

$$
\alpha \rightsquigarrow \bar{\gamma} \rightarrow \delta \rightsquigarrow \zeta \rightarrow \underline{\gamma} \rightsquigarrow \beta
$$

If

$$
p \geq n-3
$$

then $\{1,2, n-p+1, n-p+2, \ldots, n-2\} \leq \zeta$, so $G^{[p]}$ includes the path:

$$
\begin{aligned}
\alpha & \rightsquigarrow \bar{\gamma} \rightarrow \delta \rightsquigarrow\{2, n-p+1, n-p+2, \ldots, n-2, n\} \\
& \rightarrow\{1,2, n-p+1, n-p+2, \ldots, n-2\} \rightsquigarrow \zeta \rightsquigarrow \underline{\gamma} \rightsquigarrow \beta .
\end{aligned}
$$

Proceeding in this fashion we see that there is always a path from $\alpha$ to $\beta$. Thus, $G^{[p]}$ is strongly connected, so $A^{[p]}$ is irreducible.

Summarizing, for any odd $p$ we have that $A \in \mathbb{Q}^{+}$implies that $A^{[p]}$ is Metzler and irreducible. This means that (31) is a strongly cooperative dynamical system [27], and since $\Phi^{(p)}\left(t_{0}, t_{0}\right)=I$, we conclude that $\Phi^{(p)}\left(t, t_{0}\right)$ is componentwise positive for all $t>t_{0}$. In other words, all minors of order $p$ are positive for all $t>t_{0}$, so the system is CVDDS.

To prove the converse implication, assume that $A \notin \mathbb{Q}^{+}$. Then one of the following three cases holds. Case 1. The matrix $A$ is not Metzler. Then $a_{i j}<0$ for some $i \neq j$. Since $\Phi\left(t_{0}\right)=I$ and

$$
\dot{\Phi}\left(t_{0}\right)=A \Phi\left(t_{0}\right)=A
$$

it follows that entry $(i, j)$ of $\Phi\left(t_{0}+\varepsilon\right)$ is negative for all $\varepsilon>0$ sufficiently small. Thus, the system is not CVDDS.

Case 2. The matrix $A$ is Metzler, but not irreducible. In this case, it is straightforward to show that there exists an entry of $\Phi(t)$ that is zero for all $d:=t-t_{0}>0$ sufficiently small. Thus, the system is not CVDDS.

Case 3. The matrix $A$ is Metzler, irreducible, but there exist indices $w, q \in\{1, \ldots, n\}$ such that $a_{w q} \neq 0$ with

$$
1<|w-q| \text { and }|w-q|<n-1 \text {. }
$$

The first inequality here means that $a_{w q}$ is not on the main, super- or sub-diagonal. The second inequality implies that $a_{w q}$ is not entry $(1, n)$ nor $(n, 1)$. We will show that there exists an odd $p$ such $A^{[P]}$ is not Metzler. If $a_{w q}<0$ then $A^{[1]}=A$ is not Metzler. Thus, it is enough to consider the case $a_{w q}>0$. Assume, 


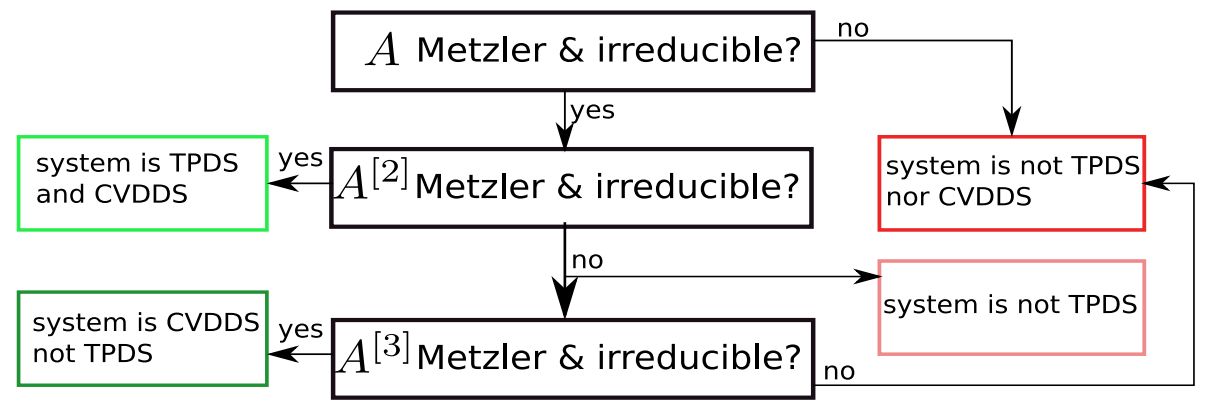

Fig. 2: Flowchart for checking if $\dot{x}(t)=A x(t)$ is CVDDS and/or TPDS.

without loss of generality, that $w<q$ (the analysis in the case $w>q$ is similar). It follows from (38) that there exists $k \in\{2, \ldots, n-1\}$ such that $w<k<q$. We consider two cases.

Case 1. Suppose that $q \neq n$. Let $\alpha:=\{w, k, n\}$ and $\beta:=\{k, q, n\}$. Then $|\alpha \cap \beta|=2$, and $i_{1}=w \neq j_{2}=q$. Thus, the entry in $A^{[3]}$ corresponding to $(\alpha, \beta)$ is $(-1)^{\ell+m} a_{i_{\ell} j_{m}}=(-1)^{1+2} a_{w q}<0$, so $A^{[3]}$ is not Metzler. Case 2. Suppose that $q=n$. Note that since $q-w<n-1, w>1$. Let $\alpha:=\{1, w, k\}$ and $\beta:=\{1, k, q\}$. Then $|\alpha \cap \beta|=2$, and $i_{2}=w \neq j_{3}=q=n$. Thus, the entry in $A^{[3]}$ corresponding to $(\alpha, \beta)$ is $(-1)^{\ell+m} a_{i_{\ell} j_{m}}=(-1)^{2+3} a_{w n}<0$, so again $A^{[3]}$ is not Metzler.

We conclude that if $A \notin \mathbb{Q}^{+}$then at least one of the matrices $A^{[1]}, A^{[3]}$ is either not Metzler or reducible. Since $\Phi\left(t_{0}\right)=I, \Phi^{(p)}\left(t_{0}\right)=I$ for all $p$. If $A^{[p]}$ is not Metzler then there exist $i, j$, with $i \neq j$, such that entry $(i, j)$ of $A^{[p]}$ is negative. Since $\dot{\Phi}^{(p)}\left(t_{0}\right)=A^{[p]} \Phi^{(p)}\left(t_{0}\right)=A^{[p]}$, it follows that entry $(i, j)$ of $\dot{\Phi}^{(p)}\left(t_{0}\right)$ is negative and thus entry $(i, j)$ of $\Phi^{(p)}\left(t_{0}+\varepsilon\right)$ is negative for all $\varepsilon>0$ sufficiently small. Thus, the system is not CVDDS. This completes the proof of Thm. 5]

Remark 3. Thm. 5 implies in particular that if $A \in M^{+}$then $\dot{x}=A x$ is CVDDS. In fact in this case the system is also TPDS, so its transition matrix satisfies the SVDP and thus it satisfies the SCVDP. In this respect, TPDS is a special case of CVDDS.

Remark 4. The proof of Thm. 5 shows that if $A \in \mathbb{Q}^{+}$then the system is CVDDS and if $A \notin \mathbb{Q}^{+}$then at least one of $A^{[1]}=A, A^{[3]}$ is not Metzler or irreducible. Thus, for any dimension $n$ it is sufficient to verify that $A$ and $A^{[3]}$ are Metzler and irreducible in order to establish CVDDS.

Combining Remark 4 with the results in [17] yields a simple flowchart for establishing if a system $\dot{x}(t)=$ $A x(t)$ is CVDDS and/or TPDS. This is depicted in Fig. 2.

Example 9. Consider the case $A=\left\{a_{i j}\right\}_{i, j=1}^{4}$. In this case the matrices $A^{[2]}, A^{[3]}$ are given in (33) and (34) and $A^{[4]}=\operatorname{tr}(A)$. To guarantee that $A^{[1]}=A$ is Metzler, $a_{i j}$ must be nonnegative for all $i \neq j$. Now (34) shows that to guarantee that $A^{[3]}$ is Metzler, we must have $a_{13}=a_{31}=a_{24}=a_{42}=0$, so $A \in \mathbb{Q}^{+}$.

if $\dot{x}=A x$ is CVDDS then we know from Thm. 4 that for any initial condition $x(0) \neq 0$ there exists a time $T \geq 0$ such that $s_{c}^{-}(x(t))=s_{c}^{+}(x(t))$ for all $t \geq T$. However, it is important to note that this does not necessarily imply that after some finite time the state-variables do not change their sign anymore. The next example demonstrates this. 
Example 10. Consider $\dot{x}=A x$ with $n=3$ and $A=\left[\begin{array}{lll}0 & 0 & 1 \\ 1 & 0 & 0 \\ 0 & 1 & 0\end{array}\right]$. This is CVDDS as $A \in \mathbb{Q}^{+}$. The solution for $x(0)=\left[\begin{array}{lll}1 & -2 & 1\end{array}\right]^{\prime}$ is

$$
x(t)=\exp (-t / 2)\left[\begin{array}{c}
\cos (p t / 2)+p \sin (p t / 2) \\
-2 \cos (p t / 2) \\
\cos (p t / 2)-p \sin (p t / 2)
\end{array}\right],
$$

where $p:=\sqrt{3}$. This means that every $x_{i}(t)$ changes sign an unbounded number of times. Note that here $s_{c}^{-}(x(t))=s_{c}^{+}(x(t))=2$ for all $t \geq 0$.

We now turn to consider (2) (and (23)) with $A(t)$ time-varying.

E. The case $A(t)$ continuous

Suppose that $t \rightarrow A(t)$ is a continuous matrix function on a time interval $(a, b)$. To derive a necessary and sufficient condition for CVDDS, we first introduce more notation. For a matrix $Q$ we write $Q \geq 0$ $[Q \gg 0]$ if every entry of $Q$ is nonnegative [positive]. The next result provides a necessary and sufficient condition for the solution of a linear matrix differential equation to be componentwise positive for all time.

Proposition 2. Let $A(\cdot):(a, b) \rightarrow \mathbb{R}^{n \times n}$ be a continuous matrix function, and assume that $A(\tau)$ is Metzler for all $\tau \in(a, b)$. Then the following two conditions are equivalent.

(1) for any interval $[p, q]$, with $a<p<q<b$, there exists $t^{*} \in[p, q]$ such that $A\left(t^{*}\right)$ is irreducible;

(2) for any pair $\left(t_{0}, t\right)$ with $a<t_{0}<t<b$ the solution of $\dot{\Phi}(\tau)=A(\tau) \Phi(\tau)$, $\Phi\left(t_{0}\right)=I$, satisfies $\Phi(t) \gg$ 0.

Proof of Proposition 2. Throughout we will use the well-known graph-theoretic interpretation of irreducibility, namely, that a matrix $B \in \mathbb{R}_{+}^{n \times n}$ (and thus also a Metzler matrix $B$ ) is irreducible iff its associated adjacency graph is strictly connected [10, Chapter 6]. This immediately implies that if $A(\tau)$ is irreducible and $A(\cdot)$ is continuous then there exists a time interval containing $\tau$ such that $A(s)$ is irreducible for all $s$ in this time interval.

For $s \in(a, b)$, let $E_{+}(s)$ denote the edges in the adjacency matrix with positive weights at time $s$ (corresponding to positive off-diagonal entries of $A(s)$ ), and let $E_{0}(s)$ denote the edges in the adjacency matrix with zero weights at time $s$ (corresponding to zero off-diagonal entries of $A(s)$ ).

Suppose that condition (1) in Proposition 2 holds. Fix $t_{0}, t$ with $a<t_{0}<t<b$. By condition (1), there exists $t^{*} \in\left[t_{0}, t\right]$ such that $A\left(t^{*}\right)$ is irreducible. By continuity, there exists a time interval $L \subseteq\left[t_{0}, t\right]$ such that $A(s)$ is irreducible for all $s \in L$ and, furthermore, the matrix

$$
\underline{A}:=\min _{s \in L} A(s)
$$


is Metzler and irreducible. For any $\tau \in(a, b)$, let

$$
B(\tau):= \begin{cases}\underline{A}, & \tau \in L \\ A(\tau), & \text { otherwise }\end{cases}
$$

and let $\Omega$ denote the solution of $\dot{\Omega}=B \Omega$, with $\Omega\left(t_{0}\right)=I$. By known results on strongly cooperative dynamical systems (see, e.g. [17]), $\Omega(t) \gg 0$. Let $\Delta:=\Phi-\Omega$. Then

$$
\dot{\Delta}=B \Delta+(A-B) \Phi
$$

and this implies that $\Delta(t) \geq 0$, so $\Phi(t) \geq \Omega(t) \gg 0$.

To prove the converse implication, assume that condition (11) does not hold. Thus, there exist $p, q$, with $a<p<q<b$, such that $A(\tau)$ is reducible for all $\tau \in[p, q]$. By continuity, there exists $\varepsilon>0$ sufficiently small such that the edges in $E_{+}(p)$ belong to $E_{+}(s)$ for all $s \in[p, p+\varepsilon]$. Since $A(\tau)$ is reducible for all $\tau \in[p, p+\varepsilon]$, there exists a non empty set of edges that are zero for all $\tau \in[p, p+\varepsilon]$ and when these edges are zero the associated graph is not strictly connected. Hence, there exists a permutation matrix $P \in\{0,1\}^{n \times n}$ such that

$$
P A(s) P^{\prime}=\left[\begin{array}{cc}
B(s) & C(s) \\
0 & D(s)
\end{array}\right], \quad \text { for all } s \in[p, p+\varepsilon],
$$

where the 0 denotes an $(n-r) \times r$ zero matrix, with $1 \leq r \leq n-1$. This implies that condition (2) does not hold for $t_{0}=p$ and any $t \in(p, p+\varepsilon]$. This completes the proof.

We can now state the main result in this subsection. Let $\mathbb{Q} \subset \mathbb{R}^{n \times n}$ denote the set of $n \times n$ matrices that are Metzler and can have non-zero entries only on the main diagonal, super- and sub-diagonals and entries $(1, n)$ and $(n, 1)$. For example, for $n=4$ any matrix in the form:

$$
\left[\begin{array}{cccc}
* & + & 0 & 0 \\
0 & * & 0 & 0 \\
0 & 0 & * & + \\
+ & 0 & 0 & *
\end{array}\right]
$$

where $*$ denotes some value and + denotes a positive value, is in $\mathbb{Q}$.

Theorem 6. Suppose that $A(\cdot)$ is a continuous matrix function on $(a, b)$. Then the following conditions are equivalent:

(1) $A(s) \in \mathbb{Q}$ for all $s \in(a, b)$, and for any interval $[p, q]$, with $a<p<q<b$, there exists $t^{*} \in[p, q]$ such that $A\left(t^{*}\right)$ is irreducible;

(2) the system (23) is CVDDS.

Note that this generalizes Theorem 5. Indeed, if $A$ is a constant matrix then condition (11) means that $A \in Q$ and $A$ is irreducible, so $A \in \mathbb{Q}^{+}$.

Proof of Theorem 6 . If $A(s) \notin \mathbb{Q}$ for some $s \in(a, b)$ then using continuity implies that $A(\cdot) \notin \mathbb{Q}$ on a time interval that includes $s$ and arguing as in the proof of Theorem 5 implies that (23) is not CVDDS. If $A(\cdot)$ is reducible on some time interval $[p, q]$ then it follows from Proposition 2 that (23) is not CVDDS. 
To prove the converse implication, assume that condition (1) holds. Pick $\left(t_{0}, t\right)$ such that $a<t_{0}<t<b$, and an odd integer $p$. Arguing as in the proof of Theorem 5 shows that $A^{[p]}$ is also Metzler and furthermore that there exists $t^{*} \in\left[t_{0}, t\right]$ such that $A^{[p]}\left(t^{*}\right)$ is irreducible. Using Proposition 2 implies that the solution of $\dot{\Phi}^{(p)}=A^{[p]} \Phi^{(p)}$, with $\Phi^{(p)}\left(t_{0}\right)=I$, satisfies $\Phi^{(p)}(t) \gg 0$, i.e. all minors of order $p$ are positive at time $t$. Since this holds for any odd $p$, this completes the proof.

\section{F. The case $A(t)$ measurable}

In this subsection, we assume that

$$
\begin{aligned}
A:(a, b) \rightarrow \mathbb{R}^{n \times n} & \text { is a matrix of locally (essentially) } \\
& \text { bounded measurable functions. }
\end{aligned}
$$

This general case is important in the context of control systems. Indeed, consider $\dot{x}=f(x, u)$, with $u$ a control input. The associated variational equation is $\dot{z}=J(x, u) z$, where $J=\frac{\partial f}{\partial x}$ is the Jacobian of $f$. In many cases, for example when considering optimal controls, one must allow measurable controls (see, e.g. [14]) and thus $t \rightarrow J(t)$ is typically a measurable, but not necessarily continuous, matrix function.

It is well-known that (39) implies that (23) admits a unique, locally absolutely continuous, nonsingular solution for all $t \in(a, b)$ (see, e.g., [28, Appendix C]). The next result provides a sufficient condition for (2) to be CVDDS.

Theorem 7. Suppose that (39) holds with $A(t) \in \mathbb{Q}^{+}$for almost all $t \in(a, b)$. Furthermore, suppose that there exists $\delta>0$ such that all the non-zero entries of $A(t)$ are larger or equal to $\delta$ for almost all $t$. Then (23) is CVDDS.

Proof of Thm. 7. Pick an odd $p \in\{1, \ldots, n\}$. Consider the dynamics $\dot{\Phi}^{(p)}(t)=A^{[p]}(t) \Phi^{(p)}(t)$. We know from the analysis in the proof of Thm. 5 that every off diagonal entry of $A^{[p]}(t)$ is either zero or equal or larger than $\delta$ for almost all $t \in(a, b)$. To analyze $\Phi^{(p)}(t)$, pick an arbitrary $1 \leq k \leq\left(\begin{array}{l}n \\ p\end{array}\right)$, and let $u(t)$ denote the $k$ th column of $\Phi^{(p)}(t)$. Then $\dot{u}(t)=A^{[p]}(t) u(t)$, with $u\left(t_{0}\right)=e^{k}$, where $e^{k}$ is the $k$ th canonical

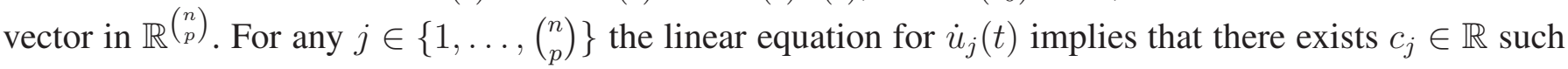
that $u_{j}(t) \geq \exp \left(c_{j}\left(t-t_{0}\right)\right) u_{j}\left(t_{0}\right)$ for all $t \geq t_{0}$. In particular, if $u_{j}(\tau)>0$ at some time $\tau$ then $u_{j}(t)>0$ for all $t \geq \tau$. Thus, $u_{k}(t)>0$ for all $t \geq t_{0}$. Pick a time $\tau \geq t_{0}$, and let $w \geq 1$ denote the number of entries $j$ such that $u_{j}(\tau)>0$. Without loss of generality, assume that these entries are $j=1,2, \ldots, w$. Write $\dot{u}=A^{[p]} u$ as

$$
\dot{u}=\left[\begin{array}{cc}
* & * \\
G & *
\end{array}\right]\left[\begin{array}{c}
u_{1} \\
\vdots \\
u_{w} \\
0
\end{array}\right],
$$

where the 0 denotes a vector of $s:=\left(\begin{array}{l}n \\ p\end{array}\right)-w$ zeros, and $G(t) \in \mathbb{R}^{s \times w}$. Since $A^{[p]}(t)$ is irreducible for almost all $t, G(t)$ has a non-zero entry that is larger or equal to $\delta$ for almost all $t$. This implies that at least $w+1$ entries in $u_{j}(t)$ are positive for all $t>\tau$. Our assumption on $A(t)$ implies that we can now use an inductive argument to conclude that all the entries of $u(t)$ are positive for all $t>t_{0}$. Since this 
holds for any odd $p$ and any index $k$, we conclude that every odd minor of $\Phi(t)$ is positive for all $t>t_{0}$. This completes the proof of Thm. 7 .

In the remainder of this section, we demonstrate how Theorem 6 can be used to show that the variational equation associated with a nonlinear dynamical model, called the ribosome flow model on a ring (RFMR), is CVDDS.

There is considerable recent interest in understanding the dynamics of mRNA translation. This is due to: (1) the introduction of several novel methods allowing to track mRNA translation in the cell [31], [11]; and (2) a growing understanding that regulation of mRNA translation plays an important role in the precise tuning of the expression of each gene in the genome, and that this is critical for many aspects of cell function [13], [18], [1].

The RFMR has been used to study the flow of ribosomes along a circular mRNA during the process of translation [21], [30], [29]. This model includes $n$ consecutive sites located along a circular ring. The normalized occupancy level (or density) of site $i$ at time $t$ is described by a state variable $x_{i}(t): \mathbb{R}_{+} \rightarrow$ $[0,1], i=1, \ldots, n$, where $x_{i}(t)=0\left[x_{i}(t)=1\right]$ means that site $i$ is completely free [full]. The transition between sites $i$ and site $i+1$ is regulated by a parameter $\lambda_{i}>0$.

From here on we interpret all indexes modulo $n$, i.e. $x_{0}=x_{n}$ and $x_{n+1}=x_{1}$. The dynamics of the RFMR is given by $n$ nonlinear first-order ordinary differential equations:

$$
\dot{x}_{i}=\lambda_{i-1} x_{i-1}\left(1-x_{i}\right)-\lambda_{i} x_{i}\left(1-x_{i+1}\right), \quad i=1, \ldots, n .
$$

This can be explained as follows. The flow of particles from site $i$ to site $i+1$ is $\lambda_{i} x_{i}(t)\left(1-x_{i+1}(t)\right)$. This flow is proportional to $x_{i}(t)$, i.e. it increases with the occupancy level at site $i$, and to $\left(1-x_{i+1}(t)\right)$, i.e. it decreases as site $i+1$ becomes fuller. Note that the maximal possible flow from site $i$ to site $i+1$ is the transition rate $\lambda_{i}$. Eq. (40) thus states that the change in the state-variable $x_{i}$ as a function of time equals the flow entering site $i$ from site $i-1$, minus the flow exiting site $i$ to site $i+1$.

It is not difficult to show that $[0,1]^{n}$ is an invariant set for the RFMR. It is easy to verify that $1_{n}$ and $0_{n}$ are equilibrium points of the RFMR (corresponding to the case where all sites are completely full and completely empty, respectively), so from here on we consider initial conditions

$$
x(0) \in[0,1]^{n} \backslash\left\{0_{n}, 1_{n}\right\} .
$$

An important property of the RFMR is that if $x_{i+1}(t) \approx 1$. i.e. site $i+1$ is "quite full" then

$$
\dot{x}_{i} \approx \lambda_{i-1} x_{i-1}\left(1-x_{i}\right) \geq 0
$$

so the density at site $i$ increases. Thus, "traffic jams" may gradually evolve behind a full site.

A calculation shows that the Jacobian $J(x)$ of the RFMR satisfies $J=M-D$, where $D:=\operatorname{diag}\left(\lambda_{n} x_{n}+\right.$ 


$$
\left.\lambda_{1}\left(1-x_{2}\right), \lambda_{1} x_{1}+\lambda_{2}\left(1-x_{3}\right), \ldots, \lambda_{n-1} x_{n-1}+\lambda_{n}\left(1-x_{1}\right)\right), \text { and }
$$

$$
M:=\left[\begin{array}{cccccc}
0 & \lambda_{1} x_{1} & 0 & 0 & \ldots & \lambda_{n}\left(1-x_{1}\right) \\
\lambda_{1}\left(1-x_{2}\right) & 0 & \lambda_{2} x_{2} & 0 & \ldots & 0 \\
0 & \lambda_{2}\left(1-x_{3}\right) & 0 & \ddots & \ldots & 0 \\
& & \ddots & & & \\
0 & \ldots & 0 & \lambda_{n-2}\left(1-x_{n-1}\right) & 0 & \lambda_{n-1} x_{n-1} \\
\lambda_{n} x_{n} & \ldots & 0 & 0 & \lambda_{n-1}\left(1-x_{n}\right) & 0
\end{array}\right] .
$$

It is straightforward to verify that if for some index $i$ we have $x_{i}(t) \equiv 0\left[x_{i}(t) \equiv 1\right]$ on a time interval then $x(t) \equiv 0_{n}\left[x(t) \equiv 1_{n}\right]$ on a time interval. Since we consider initial conditions as described in (41), we conclude that $M(x(t))$ (and thus $J(x(t))$ ) is irreducible, except perhaps at isolated points of time. Now Theorem 6 implies that the variational system $\dot{z}(t)=J(x(t)) z(t)$ is CVDDS.

\section{CONCLUSiOnS}

Several interesting studies (see e.g. [7], [25], [15]) analyzed certain types of nonlinear time-varying systems in the form $\dot{y}=f(t, y)$ by showing that the number of cyclic sign variations in the vector solution $z(t)$ of the variational system $\dot{z}(t)=J(t, y(t)) z(t)$ can only decrease with time. This was proved by direct analysis of the variational system.

Here, we developed the theoretical framework of such systems by introducing the notion of a CVDDS and analyzing its properties. A CVDDS is a linear time-varying system whose transition matrix satisfies the strong cyclic variation diminishing property. We proved that the number of cyclic sign variations in the vector solution of such a system can only decrease with time. We also derived a necessary and sufficient condition for a system to be CVDDS. This generalizes the notion of TPDS analyzed in [23], [17].

Our results suggest several interesting directions for further research. First, we believe that the systematic analysis of CVDDSs presented here can assist in developing a better understanding of what CVDDS of the variational system implies for the original nonlinear system. When the variational system $\dot{z}=J z$ is TPDS then there exists a time $T \geq 0$ such that $z(t) \in V$ for all $t \geq T$ (where $V$ is the set defined in (3)). This means in particular that either $z_{1}(t)>0$ for all $t \geq T$ or $z_{1}(t)<0$ for all $t \geq T$. Since $z_{1}=\dot{x}_{1}$, we conclude that either $x_{1}(t)$ is unbounded or it converges to a limit. Building on this, Smillie [24] and Smith [26] proved powerful stability results for time-invariant and time-varying and periodic nonlinear systems whose variational system is TPDS. However, Example 10 shows that when the variational system is CVDDS every $z_{i}(t)$ may have an unbounded number of sign variations, and thus the approach used in the TPDS case cannot be applied.

Nevertheless, the fact that $s_{c}^{-}$(and $s_{c}^{+}$) is a discrete-valued Lyapunov function certainly constraints the dynamics. For example, for a system with a compact state-space it implies that the state-space admits a Morse decomposition (see e.g. [9]). Also, there are certainly special cases where more can be said. For example, Remark 2 shows that if $s_{c}^{+}(z(t))=s_{c}^{-}(z(t))=0$ for all $t \geq T$ then necessarily $s^{+}(z(t))=$ $s^{-}(z(t))=0$, so $z(t) \in V$ for all $t \geq T$ and then we can apply the same ideas as in the TPDS case. These issues are currently under study. 
Other possible topics for further research include analyzing the spectral properties of matrices in $\mathbb{Q}^{+}$ (see e.g. [7] for some results on this topic), and studying the properties of the nonlinear $T$-periodic system $\dot{y}=f(t, y)$ satisfying $J(t, y) \in \mathbb{Q}^{+}$for all $t$.

\section{ACKNOWLEDGMENTS}

$\mathrm{MM}$ is grateful to Juergen Garloff for bringing to his attention the work in Chapter 5 of [12] and to Rola Alsaidi for helpful comments.

\section{BIOGRAPHIES}

Tsuff Ben Avraham is an undergraduate student in the EE-Physics program at Tel Aviv University, Israel. Guy Sharon is an undergraduate student in the EE-Physics program at Tel Aviv University, Israel. Yoram Zarai received the BSc (cum laude), $\mathrm{MSc}$ and $\mathrm{PhD}$ degrees in EE from Tel Aviv University, in 1992, 1998 and 2016 respectively. He is currently a postdoctoral fellow at the Faculty of Engineering, Tel Aviv University. His research interests include modeling and analysis of biological phenomena, systems and control theory and machine learning.

Michael Margaliot received the BSc (cum laude) and MSc degrees in EE from the Technion-Israel Institute of Technology-in 1992 and 1995, respectively, and the $\mathrm{PhD}$ degree (summa cum laude) from Tel Aviv University in 1999. He was a post-doctoral fellow in the Dept. of Theoretical Math. at the Weizmann Institute of Science. In 2000, he joined the Department of EE-Systems, Tel Aviv University, where he is currently a Professor and Chair. His research interests include the stability analysis of differential inclusions and switched systems, optimal control theory, fuzzy control, computation with words, Boolean control networks, contraction theory, cooperative systems, and systems biology. He is coauthor of New Approaches to Fuzzy Modeling and Control: Design and Analysis, World Scientific, 2000 and of Knowledge-Based Neurocomputing, Springer, 2009. He served as an Associate Editor for the journal IEEE Transactions on Automatic Control during 2015 - 2017.

\section{REFERENCES}

[1] A. Bastide and A. David, "The ribosome, (slow) beating heart of cancer (stem) cell," Oncogenesis, vol. 7, 2018.

[2] L. O. Chua and T. Roska, "Stability of a class of nonreciprocal cellular neural networks," IEEE Trans. Circuits and Systems, vol. 37, no. 12, pp. 1520-1527, 1990.

[3] P. Donnell, S. A. Baigent, and M. Banaji, "Monotone dynamics of two cells dynamically coupled by a voltage-dependent gap junction," J. Theoretical Biology, vol. 261, no. 1, pp. 120-125, 2009.

[4] S. M. Fallat and C. R. Johnson, Totally Nonnegative Matrices. Princeton, NJ: Princeton University Press, 2011.

[5] C. Fang, M. Gyllenberg, and Y. Wang, "Floquet bundles for tridiagonal competitive-cooperative systems and the dynamics of timerecurrent systems," Siam J. Math. Anal., vol. 45, no. 4, pp. 2477-2498, 2013.

[6] M. Fiedler, Special Matrices and Their Applications in Numerical Mathematics, 2nd ed. Mineola, NY: Dover Publications, 2008.

[7] G. Fusco and W. M. Oliva, "Transversality between invariant manifolds of periodic orbits for a class of monotone dynamical systems," J. Dyn. Differ. Equ., vol. 2, no. 1, pp. 1-17, 1990.

[8] F. R. Gantmacher and M. G. Krein, Oscillation Matrices and Kernels and Small Vibrations of Mechanical Systems. Providence, RI: American Mathematical Society, 2002, translation based on the 1941 Russian original.

[9] T. Gedeon, Cyclic Feedback Systems, ser. Memoirs of the American Mathematical Society. Boston, MA: American Mathematical Society, 1998, vol. 637.

[10] R. A. Horn and C. R. Johnson, Matrix Analysis, 2nd ed. Cambridge University Press, 2013.

[11] N. T. Ingolia, "Ribosome footprint profiling of translation throughout the genome," Cell, vol. 165, no. 1, pp. 22-33, 2016. 
[12] S. Karlin, Total Positivity, Volume 1. Stanford, CA: Stanford University Press, 1968.

[13] E. Korkmazhan, H. Teimouri, N. Peterman, and E. Levine, "Dynamics of translation can determine the spatial organization of membranebound proteins and their mRNA," Proceedings of the National Academy of Sciences, vol. 114, pp. 13 424-13 $429,2017$.

[14] D. Liberzon, Calculus of Variations and Optimal Control Theory: A Concise Introduction. Princeton, NJ: Princeton University Press, 2012.

[15] J. Mallet-Paret and H. L. Smith, "The Poincare-Bendixson theorem for monotone cyclic feedback systems," J. Dyn. Differ. Equ., vol. 2, no. 4, pp. 367-421, 1990.

[16] M. Margaliot and T. Tuller, "Stability analysis of the ribosome flow model," IEEE/ACM Trans. Comput. Biol. Bioinf., vol. 9, pp. 1545-1552, 2012.

[17] M. Margaliot and E. D. Sontag, "Revisiting totally positive differential systems: A tutorial and new results," 2018, submitted. [Online]. Available: https://arxiv.org/abs/1802.09590

[18] E. W. Mills and R. Green, "Ribosomopathies: There's strength in numbers," Science, vol. 358, no. 6363, 2017.

[19] J. S. Muldowney, "Compound matrices and ordinary differential equations," The Rocky Mountain J. Math., vol. 20, no. 4, pp. 857-872, 1990.

[20] A. Pinkus, Totally Positive Matrices. Cambridge, UK: Cambridge University Press, 2010.

[21] A. Raveh, Y. Zarai, M. Margaliot, and T. Tuller, "Ribosome flow model on a ring," IEEE/ACM Trans. Comput. Biol. Bioinf., vol. 12, no. 6, pp. 1429-1439, 2015.

[22] I. J. Schoenberg and A. Whitney, "A theorem on polygons in $n$ dimensions with applications to variation-diminishing and cyclic variation-diminishing linear transformations," Compositio Math., vol. 9, pp. 141-160, 1951.

[23] B. Schwarz, "Totally positive differential systems," Pacific J. Math., vol. 32, no. 1, pp. 203-229, 1970.

[24] J. Smillie, "Competitive and cooperative tridiagonal systems of differential equations," SIAM J. Mathematical Analysis, vol. 15, pp. $530-534,1984$.

[25] H. L. Smith, “A discrete Lyapunov function for a class of linear differential equations,” Pacific J. Math., vol. 144, no. 2, pp. 345-360, 1990.

[26] H. L. Smith, "Periodic tridiagonal competitive and cooperative systems of differential equations," SIAM J. Math. Anal., vol. 22, no. 4, pp. 1102-1109, 1991.

[27] H. L. Smith, Monotone Dynamical Systems: An Introduction to the Theory of Competitive and Cooperative Systems, ser. Mathematical Surveys and Monographs. Providence, RI: Amer. Math. Soc., 1995, vol. 41.

[28] E. D. Sontag, Mathematical Control Theory: Deterministic Finite Dimensional Systems, 2nd ed. New York: Springer, 1998.

[29] Y. Zarai, M. Margaliot, and T. Tuller, "On the ribosomal density that maximizes protein translation rate," PLOS ONE, vol. 11, no. 11, pp. 1-26, 2016.

[30] Y. Zarai, A. Ovseevich, and M. Margaliot, “Optimal translation along a circular mRNA," Sci. Rep., vol. 7, no. 1, p. $9464,2017$.

[31] E. Zlotorynski, "Live stream: translation at single-mRNA resolution," Nature Reviews Molecular Cell Biology, vol. 17, p. $395,2016$. 